\title{
Hippocampal-Prefrontal Reactivation during Learning Is Stronger in Awake Compared with Sleep States
}

\author{
Wenbo Tang, ${ }^{1}$ Justin D. Shin, ${ }^{1}{ }^{\circ}$ Loren M. Frank, ${ }^{2}$ and ${ }^{-S h a n t a n u ~ P . ~ J a d h a v ~}{ }^{1,3}$ \\ ${ }^{1}$ Graduate Program in Neuroscience, Brandeis University, Waltham, Massachusetts, 02453, ${ }^{2}$ Howard Hughes Medical Institute, Department of Physiology \\ and Kavli Institute for Fundamental Neuroscience, University of California, San Francisco, California, 94143, and ${ }^{3}$ Neuroscience Program, Department of \\ Psychology and Volen National Center for Complex Systems, Brandeis University, Waltham, Massachusetts, 02453
}

\begin{abstract}
Hippocampal sharp-wave ripple (SWR) events occur during both behavior (awake SWRs) and slow-wave sleep (sleep SWRs). Awake and sleep SWRs both contribute to spatial learning and memory, thought to be mediated by the coordinated reactivation of behavioral experiences in hippocampal-cortical circuits seen during SWRs. Current hypotheses suggest that reactivation contributes to memory consolidation processes, but whether awake and sleep reactivation are suited to play similar or different roles remains unclear. Here we addressed that issue by examining the structure of hippocampal (area CA1) and prefrontal (PFC) activity recorded across behavior and sleep stages in male rats learning a spatial alternation task. We found a striking state difference: prefrontal modulation during awake and sleep SWRs was surprisingly distinct, with differing patterns of excitation and inhibition. CA1-PFC synchronization was stronger during awake SWRs, and spatial reactivation, measured using both pairwise and ensemble measures, was more structured for awake SWRs compared with post-task sleep SWRs. Stronger awake reactivation was observed despite the absence of coordination between network oscillations, namely hippocampal SWRs and cortical delta and spindle oscillations, which is prevalent during sleep. Finally, awake CA1-PFC reactivation was enhanced most prominently during initial learning in a novel environment, suggesting a key role in early learning. Our results demonstrate significant differences in awake and sleep reactivation in the hippocampal-prefrontal network. These findings suggest that awake SWRs support accurate memory storage and memory-guided behavior, whereas sleep SWR reactivation is better suited to support integration of memories across experiences during consolidation.
\end{abstract}

Key words: hippocampus; prefrontal cortex; reactivation; sharp-wave ripple; sleep; spatial learning

\section{Significance Statement}

Hippocampal sharp-wave ripples (SWRs) occur both in the awake state during behavior and in the sleep state after behavior. Awake and sleep SWRs are associated with memory reactivation and are important for learning, but their specific memory functions remain unclear. Here, we found profound differences in hippocampal-cortical reactivation during awake and sleep SWRs, with key implications for their roles in memory. Awake reactivation is a higher-fidelity representation of behavioral experiences, and is enhanced during early learning, without requiring coordination of network oscillations that is seen during sleep. Our findings suggest that awake reactivation is ideally suited to support initial memory formation, retrieval and planning, whereas sleep reactivation may play a broader role in integrating memories across experiences during consolidation.

\section{Introduction}

The hippocampus and prefrontal cortex (PFC) are required for encoding, storage, and retrieval of memories, and play a key role

\footnotetext{
Received Aug. 13, 2017; revised Sept. 26, 2017; accepted 0ct. 22, 2017.

Author contributions: L.M.F. and S.P.J. designed research; W.T., J.D.S., and S.P.J. performed research; W.T. and S.P.J. analyzed data; W.T., L.M.F., and S.P.J. wrote the paper.

This work was supported by NIH Grants R00 MH100284 and R01 MH112661, a Sloan Research Fellowship in Neuroscience (Alfred P. Sloan Foundation), a NARSAD Young Investigator Grant (Brain and Behavior Foundation), and Whitehall Foundation award to S.P.J.; Training Grants R90 DA033463 to W.T. and T32 MH1992922 to J.D.S.; and HHMI support and NIH R01 MH105174 to L.M.F.

The authors declare no competing financial interests.

Correspondence should be addressed to Dr. Shantanu P. Jadhav, Brandeis University, 415 South Street, MS 062, Waltham, MA 02453. E-mail: shantanu@brandeis.edu.
}

in using past experiences to guide behavior (Eichenbaum and Cohen, 2001; Tse et al., 2011; Shin and Jadhav, 2016). The physiological mechanisms that mediate hippocampal-prefrontal interactions, and the specific role they play in memory, are therefore of great interest. One prominent network pattern that mediates hippocampal-prefrontal interactions is hippocampal sharp-wave ripples (SWRs; or ripples), high-frequency $(150-250 \mathrm{~Hz})$ transient oscillations ( $\sim 100 \mathrm{~ms}$ ) seen during slow-wave sleep (SWS), and in the awake state during consummation and immobility 
(Battaglia et al., 2011; Carr et al., 2011; Buzsáki, 2015; Colgin, 2016; Shin and Jadhav, 2016). These highly synchronized events are associated with reactivation of behavioral experiences; fast time scale replay of hippocampal place cell activity (Wilson and McNaughton, 1994; Foster and Wilson, 2006; O'Neill et al., 2008; Karlsson and Frank, 2009), which is coordinated with cortical activity (Ji and Wilson, 2007; Peyrache et al., 2009; Wierzynski et al., 2009; Jadhav et al., 2016; Wang and Ikemoto, 2016). SWR reactivation during both sleep and waking states is thought to play critical roles in memory.

Sleep reactivation is hypothesized to support memory consolidation by strengthening hippocampal-cortical memory traces (Buzsáki, 1996; Sutherland and McNaughton, 2000; Inostroza and Born, 2013). During SWS, hippocampal SWRs are coordinated with cortical spindles $(12-18 \mathrm{~Hz})$ and delta $(1-4 \mathrm{~Hz})$ oscillations, causing widespread modulation throughout the brain (Siapas and Wilson, 1998; Sirota et al., 2003; Logothetis et al., 2012; Inostroza and Born, 2013; Staresina et al., 2015). This coordination is thought to be important for consolidation; disrupting sleep SWRs impairs incremental spatial learning (Girardeau et al., 2009; Ego-Stengel and Wilson, 2010), whereas memory is enhanced by boosting coordinated oscillations and reactivation (Marshall et al., 2006; Rasch et al., 2007; Bendor and Wilson, 2012; Maingret et al., 2016). Hippocampal-prefrontal coordination, in particular, is thought to be crucial for long-term memory storage and also schema formation by integrating across experiences (Wiltgen et al., 2004; Tse et al., 2011; Genzel et al., 2014). Indeed, reactivation of behavioral experiences during sleep is observed in prefrontal ensembles, and coincides with hippocampal SWRs (Euston et al., 2007; Peyrache et al., 2009).

Awake reactivation, on the other hand, has been hypothesized to support not just consolidation, but also retrieval and memoryguided decision-making (O'Neill et al., 2010; Carr et al., 2011). Awake SWRs occur prominently during pauses in exploratory behavior, are upregulated by novelty and reward, and associated hippocampal replay events continually reactivate ongoing behavioral experiences from immediate past as well as upcoming trajectories and novel shortcut sequences (Foster and Wilson, 2006; Cheng and Frank, 2008; Karlsson and Frank, 2009; Singer and Frank, 2009; Gupta et al., 2010; Pfeiffer and Foster, 2013). This proposed role of awake SWRs in reinforcement learning, retrieval, and prospective decision-making suggests prefrontal involvement (Yu and Frank, 2015; Shin and Jadhav, 2016). In support of this possibility, we have previously shown that awake SWRs mediate coordinated hippocampal-prefrontal reactivation (Jadhav et al., 2016), and disrupting awake SWRs impairs spatial learning that requires integration across space and time (Jadhav et al., 2012).

The importance of awake and sleep SWRs in learning and memory-guided behavior is thus well established, but the specific role that each type of reactivation plays remains unclear. More structured hippocampal reactivation in awake compared with rest periods has been observed (Karlsson and Frank, 2009; Grosmark and Buzsáki, 2016), but activity outside the hippocampus and changes over learning have not been studied. Broadly, despite the differences in neuromodulatory tone between waking and sleep (Diekelmann and Born, 2010) and the different behavioral and internal contexts (Carr et al., 2011; Roumis and Frank, 2015), hippocampal-cortical reactivation in the two states during learning has not been directly compared. Here, we show that hippocampal-prefrontal reactivation during awake and sleep
SWRs is distinct, and awake reactivation is stronger and enhanced especially during initial learning.

\section{Materials and Methods}

Animals and experimental design. Five male Long-Evans rats (RRID: RGD_2308852) weighing 450-550 g were used in this study. All procedures were conducted in accordance with the guidelines of the National Institutes of Health and approved by the Institutional Animal Care and Use Committees at University of California San Francisco and Brandeis University. Animals were kept on a $12 \mathrm{~h}$ regular light/dark cycle. After several weeks of habituation to daily handling, animals were pretrained to seek liquid food rewards (sweetened evaporated milk) on an elevated linear track as described previously (Jadhav et al., 2012). After animals alternated on the linear track reliably, they were chronically implanted with a multi-tetrode drive (see Surgical implantation and electrophysiol$o g y$ ). Following recovery from the implantation surgery ( $\sim 5-7 \mathrm{~d})$, animals were food-deprived to maintain $85-90 \%$ of their ad libitum weight and again pretrained to run on a linear track. At $\sim 14 \mathrm{~d}$ after implantation, animals were introduced to the $\mathrm{W}$-track for the first time when the recording started, and gradually learned the task rules to get rewards over multiple training sessions (see Behavioral task). Following the conclusion of the experiments, we made microlesions through each electrode tip to mark recording locations.

Behavioral task. Rats learned a W-track spatial task over multiple training sessions either across $5 \mathrm{~d}$ ( $n=3$ rats, multiday animals), or within a single day ( $n=2$ rats, single-day animals). Figure 1 shows the behavior paradigm and experimental design. All animals ran 15-20 min sessions on a W-track interleaved with $30-40 \mathrm{~min}$ rest sessions in a sleep box. For the multiday training (Jadhav et al., 2016), animals performed two run sessions per day for $5 \mathrm{~d}$. Data from the behavioral periods for these three multiday animals has been presented previously to demonstrate coordinated reactivation of hippocampal (area CA1)-PFC behavioral activity during awake SWRs (Jadhav et al., 2016). The first and last rest sessions of each day were used as pre-task sleep and post-task sleep, respectively. All results presented in the paper remained unchanged using data from just these multiday animals (data not shown). For the single-day training, animals performed $8-12$ run sessions interleaved with rest sessions. The first three rest sessions were used as pre-task sleep, and the last two rest sessions were used as post-task sleep. The rest sessions for single-day animals were chosen to provide sufficiently long periods of SWS (for the durations of each sleep state, see Table 2), and we found similar results with different choices of sessions for pre-task and post-task sleep (see Fig. $4 \mathrm{H}$, where awake reactivation is compared with sleep reactivation in rest sessions that occur both before and after the run session).

The W-tracks had dimensions of $\sim 80 \times 80 \mathrm{~cm}$ with $\sim 7$-cm-wide track sections. Three reward food wells were located at the end of three arms of the W-track. Evaporated milk rewards were automatically delivered in the food wells triggered by crossing of an infrared beam by the rat's nose. Rats had to learn an alternation strategy for rewards (Fig. 1A): returning to the center well after visits to either side well (inbound component), and for choosing the opposite side well from the previous side trajectory when starting from the center well (outbound component). During rest sessions, the rat remained undisturbed in a high-walled black box $(\sim 30 \times 30 \mathrm{~cm})$, in which animals often slept.

To evaluate learning effects (see Fig. 8), behavioral data were divided into four performance categories based on subjects' raw outbound performance (proportion correct; Singer et al., 2013). These categories separated sessions into periods of (1) the first exposure to the task, (2) initial learning as the first session that animals performed above chance level of 0.5 , (3) early learning performance as the first session that animals performed $>75 \%$ correct, and (4) well learned performance as the last session animals performed $>75 \%$ correct. For visualization purposes, we used a state-space model of learning to estimate animals' performance as previously described (Jadhav et al., 2012; see Fig. 7A). All five rats performed $>75 \%$ correct in the $\mathrm{W}$-track task toward the end of learning (see Figs. 7A, 8A).

Surgical implantation and electrophysiology. Surgical implantation procedures were as previously described (Jadhav et al., 2012, 2016). Animals were implanted with a microdrive array with either 21 (multiday ani- 
A

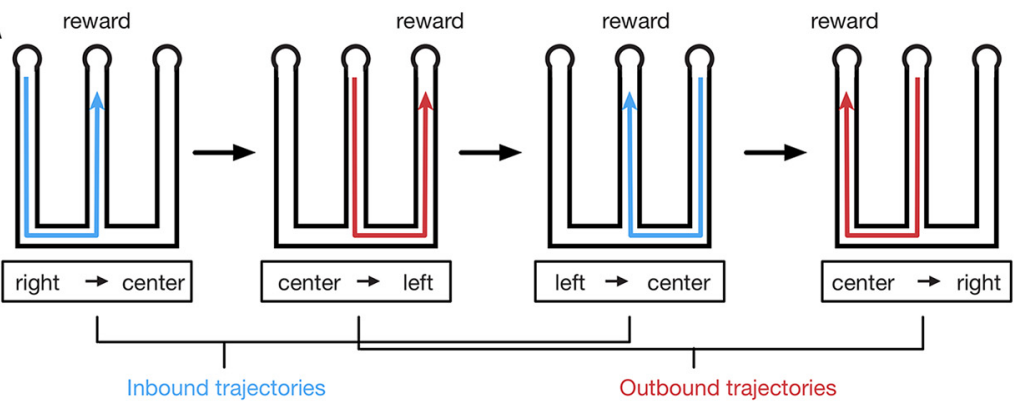

B
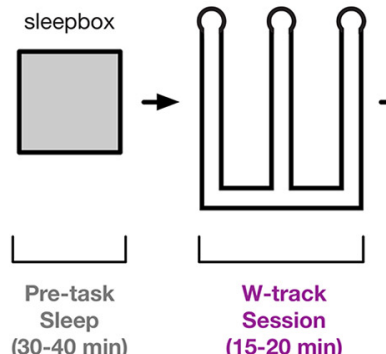

(15-20 min)
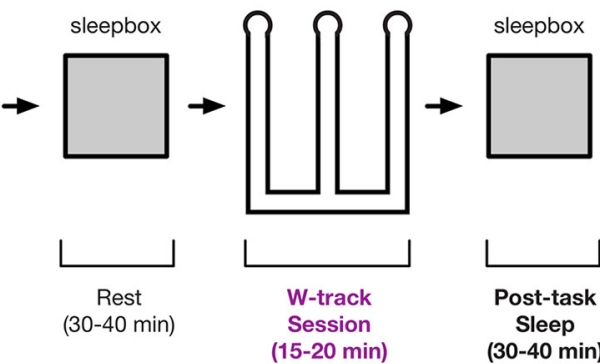

C Multi-day learning timeline ( $n=3$ rats)
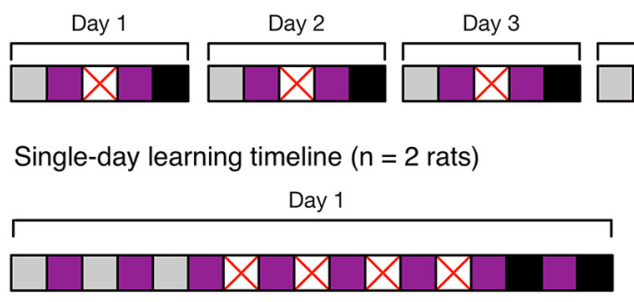

Day 4

Single-day learning timeline ( $n=2$ rats)
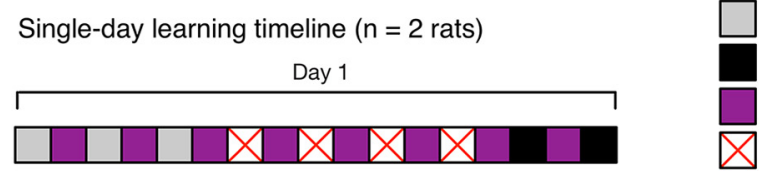

pre-task sleep

post-task sleep

W-track

Rest (not used)

D

REM boundary
PFC spike
CA1-pyr spike
SWS
REM
$-\quad$ theta:delta
theta:delta threshold

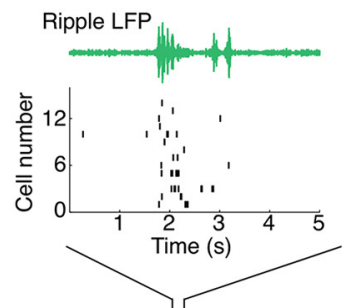

Ripple LFP (150 - $250 \mathrm{~Hz})$
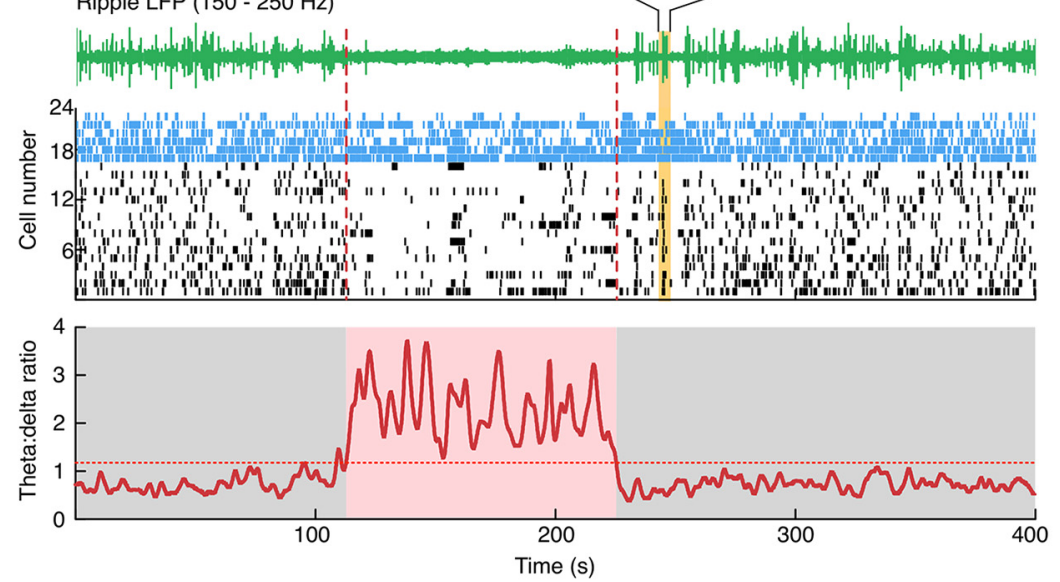

Figure 1. Experimental design and neural activity patterns during sleep. $\boldsymbol{A}, \mathrm{W}$-track spatial alternation task. Animals run in a W-shaped maze with a center arm and two outer arms, and learn to alternate between these three arms to get reward (see Materials and Methods). $\boldsymbol{B}$, Schematic of one recording block, including 15-20 min W-track sessions, interleaved with 30-40 min rest sessions in the sleep box. C, Recording timelines for multiday training (top) and single-day training (bottom). All animals ( $n=$ 5 rats) were naive to the novel W-track before training. For multiday training, animals $(n=3)$ performed two run sessions per day, and the first and last rest sessions of each day were used as pre-task sleep and post-task sleep. For single-day training, animals $(n=2)$ learned the task over eight run sessions within a single day, for which the first three rest sessions were used as pre-task mals) or 32 (single-day animals) independently moveable tetrodes at the following coordinates: right dorsal hippocampal region CA1 ( $-3.6 \mathrm{~mm}$ AP and $2.2 \mathrm{~mm} \mathrm{ML})$, right PFC (+3.0 $\mathrm{mm} \mathrm{AP}$ and $0.7 \mathrm{~mm} \mathrm{ML})$, and intermediate CA1 ( $-6.3 \mathrm{~mm}$ AP and $5.5 \mathrm{~mm} \mathrm{ML}$, in 2 multiday animals).

On the days following surgery, hippocampal tetrodes were gradually advanced to the desired depths. Hippocampal cell layer was identified by characteristic EEG patterns (sharp wave polarity, theta modulation) and neural firing patterns as previously described (Jadhav et al., 2012, 2016). For multiday animals, tetrode positions were adjusted after each day's recordings. For each animal, one tetrode in corpus callosum served as hippocampal reference (REF) tetrode, and another tetrode in overlying cortical regions served as prefrontal REF tetrode. The reference tetrodes were also referenced to a ground (GND) screw installed in skull overlying cerebellum.

Electrophysiological recordings were performed using either an NSpike system (L.M.F. and J. MacArthur, Harvard Instrumentation Design Laboratory, Cambridge, MA; for multiday animals) or a SpikeGadgets system (for single-day animals). Spike data were sampled at $30 \mathrm{kHz}$ and bandpass filtered between 300 or $600 \mathrm{~Hz}$ and $6 \mathrm{kHz}$. Local field potentials (LFPs) were sampled at $1.5 \mathrm{kHz}$ and bandpass filtered between 0.5 and $400 \mathrm{~Hz}$. During recordings, the animal's position and speed were recorded with an overhead monochrome CCD camera (30 fps) and tracked by the LEDs affixed to the headstage. Spikes were sorted as previously described (Jadhav et al., 2012, 2016). In brief, single units were identified by clustering spikes using peak amplitude, principal components, and spike width (MatClust, M. P. Karlsson). Only well isolated neurons with stable spiking waveforms were included.

Unit inclusion. Units included in analyses fired at least 100 spikes in each session. Putative interneurons were identified on the basis of average firing rate and/or spike width as previously described (Jadhav et al., 2016) and were excluded from all analyses. A peak rate was de-

$\leftarrow$

sleep, and the last two rest sessions were used as post-task sleep. The rest sessions for single-day animals were chosen to provide sufficiently long periods of SWS (Table 2), and we found similar results using only the multiday animals (data not shown). D, CA1 and PFC neuronal firing patterns in REM and SWS stages. SWS and REM stages were segregated using the ratio of theta and delta power (bottom). Simultaneously recorded spiking activity of PFC (blue ticks) and CA1 (black ticks) neurons is shown along with ripple-filtered CA1 LFP (green; $150-250 \mathrm{~Hz}$ ). REM stage is characterized by the enhanced theta power and associated theta-modulated firing of CA1 pyramidal cells (note enhanced theta- delta ratio in the bottom plot, and horizontal stripes during REM in the CA1 raster plot). In SWS stage, characteristic large amplitude ripples (in ripplefiltered LFPs) are observed, coincident with bursts of activity of the CA1 pyramidal cells (note the vertical stripes in the raster plot). Top, An expanded example of (A1 bursting events during SWRs. 

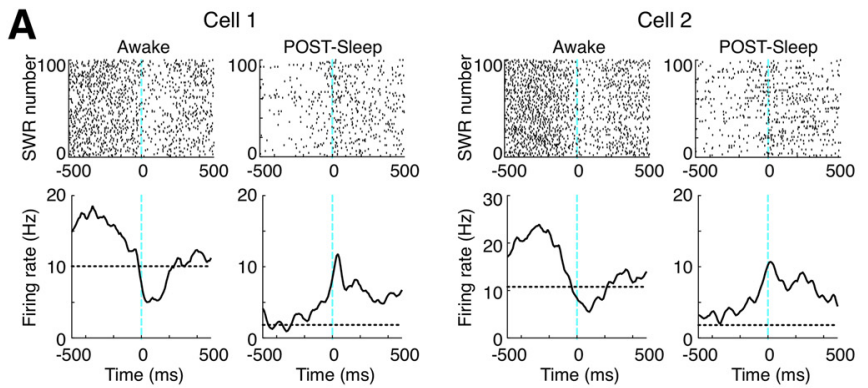

B (i) Awake (sorted) (ii)POST-Sleep (iii)POST-Sleep (sorted)

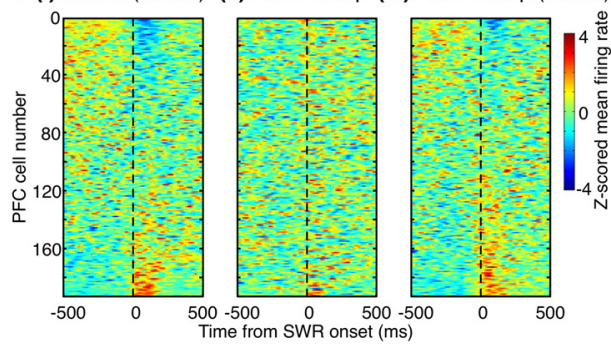

$\mathbf{F}$

G
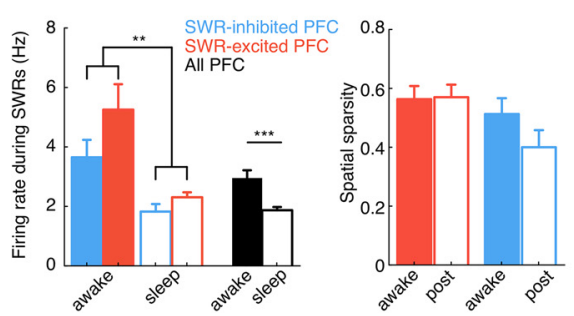

H

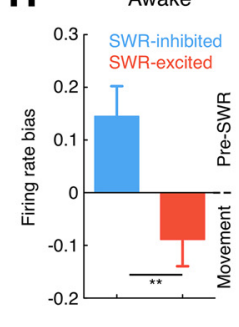

C

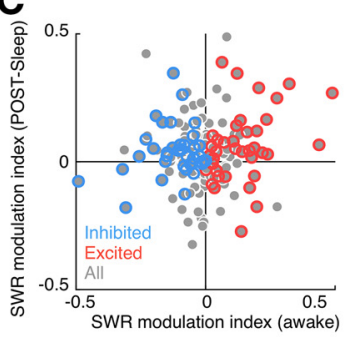

Cell 3
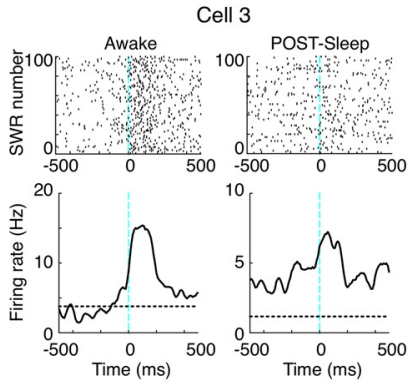

D
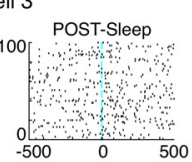

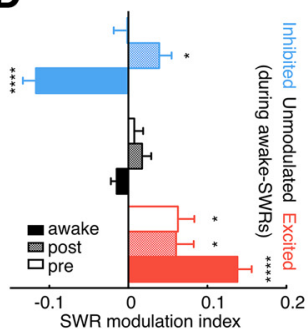

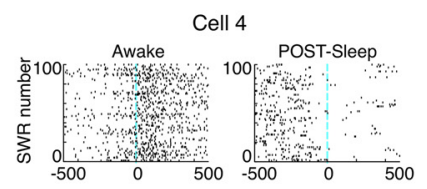

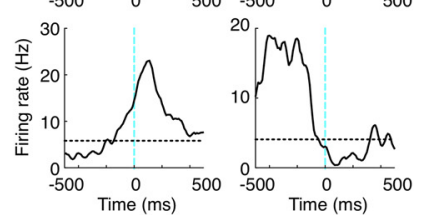

E
I

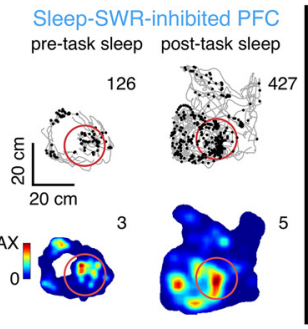

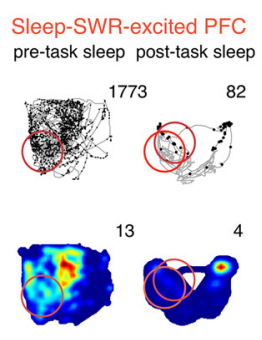

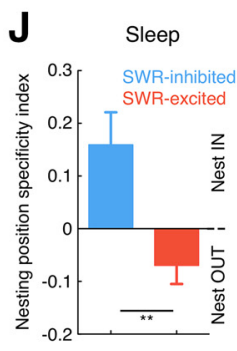

Figure 2. PFC modulation differs for awake and sleep hippocampal SWRs. A, CA1 SWR-aligned raster plots (top) and corresponding PSTHs (bottom) of four example PFC neurons showing differing modulation. Awake-SWR-inhibited neurons (Cells 1 and 2) show significant excitation during sleep SWRs. The responses of the two awake-SWR-excited neurons (Cells 3 and 4) become either less modulated (Cell 3) or inhibited (Cell 4) during sleep SWRs. Horizontal dashed lines: mean firing rates in the entire session. POST-Sleep, Post-task sleep. $B, Z$-scored SWR-triggered PSTHs of all PFC cells during awake SWRs and post-task sleep SWRs. Each row represents one PFC cell. Note that the data from the same PFC neurons are plotted in $\boldsymbol{B i}-\boldsymbol{B i i i}$. Neurons in $\boldsymbol{B i}$ and $\boldsymbol{B i i}$ are sorted by their mean firing rates in a $0-200 \mathrm{~ms}$ window after awake SWR onset, whereas neurons in $\boldsymbol{B}$ iii are sorted by their mean firing rates in a $0-200 \mathrm{~ms}$ window after post-task sleep SWR onset. C, Modulation of PFC responses during awake SWRs and sleep SWRs is not correlated $(n=193$ cells; $r=0.029, p=0.68$, Spearman correlation). Colors indicate the types/categories of modulation during awake SWRs. D, SWR modulation indexes of PFC cells belonging to different categories of awake-SWR-modulated populations are shown during pre-task sleep (open bars), wakefulness (solid bars), and post-task sleep (hatched bars). Note that awake SWR-inhibited cells (blue bars) become significantly excited during post-task sleep SWRs, but not pre-task sleep SWRs ( ${ }^{*} p<0.05,{ }^{* * * *} p<0.0001$; rank sum test for differences from 0 ). E, Population-averaged SWR-triggered PSTHs for awake-SWR-inhibited cells $(n=38)$ during pre-task sleep SWRs (bottom left), awake SWRs (top right), and post-task sleep SWRs (bottom right). Shadings, SEM; vertical dashed lines, SWR onset; horizontal dashed lines, zero (Z-score). $\boldsymbol{F}$, Mean firing rates of PFC cells during awake and sleep SWRs. Firing rates were overall higher during awake SWRs than sleep SWRs $\left({ }^{* * *} p=0.0003\right.$, Wilcoxon signed rank paired test), but importantly, firing rates were similar for SWR-inhibited versus SWR-excited PFC cells during both awake and sleep SWRs $\left({ }^{* *} p=0.0038\right.$; Kruskal-Wallis test, Dunn's post hoc). G, Spatial sparsity does not differ among different SWR-modulated PFC populations ( $n=39,47,38$, and 20 cells, respectively, for awake-SWR-excited, POST-SWR-excited, awake-SWR-inhibited, and POST-SWR-inhibited cells), illustrating no difference between basic spatial coding properties of SWR-inhibited and SWR-excited neurons in awake and sleep states ( $p=0.21$, Kruskal-Wallis test). Spatial sparsity is calculated as a fraction of linearized place field with a firing rate $>25 \%$ of its peak rate (Jadhav et al., 2016). $\mathbf{H}$, Immobility coding of awake-SWR-modulated PFC cells, showing the firing rate bias quantifying difference during pre-SWR periods and high-speed $(>10 \mathrm{~cm} / \mathrm{s})$ periods. Note that compared with awake-SWR-excited cells, awake-SWR-inhibited cells were more active during periods of immobility when SWRs are prominent, as reported previously (Jadhav et al., 2016). ${ }^{* *} p=0.004$, rank sum test. $I, J$, Immobility coding of sleep-SWR-modulated PFC cells. I, Examples of spatial firing maps in the sleep box of two sleep-SWR-inhibited (left) and two sleep-SWR-excited (right) PFC cells. Data from waking periods in the box is plotted. Top, The gray lines represent all the visited locations, and the black dots indicate the positions where the cell fired. Numbers on top right of each plot denote total number of spikes. Bottom, Corresponding spatial firing maps. Numbers on top right of each plot denote peak spatial firing rate. Each red circle indicates the nesting position ( $5 \mathrm{~cm}$ radius) of the animal for a sleep period ( $>5 \mathrm{~min}$ ) detected in the same recording session. The difference in the firing rates within (Nest IN) and outside (Nest OUT) the encircled regions was computed as the specificity index, varying between -1 and 1 , as previously described (Kay et al., 2016). Note the enhanced Nest-IN firing of sleep-SWR-inhibited PFC cells. J, Nesting position specificity indexes in sleep-SWR-excited ( $n=51$ ) and -inhibited ( $n=24)$ PFC populations. Only cells recorded from a rest session with at least one sleep period $>5$ min (determined by the animal's head speed; see Materials and Methods) are included. Note that sleep-SWR-inhibited cells showed significantly stronger Nest-IN firing than sleep-SWR-excited cells. ${ }^{* *} p=0.005$, rank sum test.

fined as the maximum rate across all spatial bins in the linearized spatial map (see Spatial maps). A peak rate of $3 \mathrm{~Hz}$ or greater was required for a cell to be considered a place cell. For across-session comparisons (Fig. 2), only cells that were recorded continuously across all these sessions with stable spiking waveforms were included, unless otherwise specified. Table 1 shows the distribution of cells across animals. All our results were similar with exclusion of intermediate CA1 cells $(n=31$ of total 346 CA1 cells; data not shown).
Sleep state identification. Animals' head speed, hippocampal, and prefrontal LFPs were used to classify sleep states into NREM/SWS and REM sleep periods using established methods (Mizuseki et al., 2011; Kay et al., 2016; Fig. 1D). In the sleep box, awake periods were identified as times with head speed $>4 \mathrm{~cm} / \mathrm{s}$ (threshold speed), in addition to times $<4 \mathrm{~cm} / \mathrm{s}$ within $7 \mathrm{~s}$ after active moving (speed greater than threshold). Candidate sleep periods were identified as times with head speed $<4 \mathrm{~cm} / \mathrm{s}$ preceded by a $1 \mathrm{~min}$ immobility period (speed less than threshold). SWS/NREM 
Table 1. Cell distribution across animals

\begin{tabular}{|c|c|c|c|c|c|c|c|c|}
\hline \multirow[b]{3}{*}{ Animal } & \multirow[b]{3}{*}{ CA1 cells } & \multicolumn{7}{|c|}{ PFC cells } \\
\hline & & \multirow[b]{2}{*}{ All } & \multicolumn{3}{|c|}{ SWR-excited } & \multicolumn{3}{|c|}{ SWR-inhibited } \\
\hline & & & PRE & AWAKE & POST & PRE & AWAKE & POST \\
\hline $\mathrm{HPa}$ & 99 & 37 & 12 & 11 & 13 & 2 & 11 & 1 \\
\hline $\mathrm{HPb}$ & 131 & 69 & 18 & 12 & 17 & 7 & 8 & 4 \\
\hline $\mathrm{HPC}$ & 61 & 42 & 1 & 5 & 3 & 4 & 5 & 5 \\
\hline ER1 & 22 & 21 & 5 & 7 & 8 & 2 & 8 & 5 \\
\hline KL8 & 33 & 24 & 3 & 4 & 6 & 3 & 6 & 5 \\
\hline Total & 346 & 193 & 39 & 39 & 47 & 18 & 38 & 20 \\
\hline
\end{tabular}

Summary of the number of CA1 and PFC excitatory cells recorded from each animal. Only the cells present across all analyzed sessions per day and meeting the inclusion criteria (see Materials and Methods) are reported here. PRE, Pre-task sleep session; AWAKE, W-track session; POST, post-task sleep session.

and REM were further separated within candidate sleep periods. Briefly, theta $(6-12 \mathrm{~Hz})$ and delta $(1-4 \mathrm{~Hz})$ power was bandpass filtered and averaged from all available CA1 tetrodes (referenced to GND). A threshold (mean $+1 \mathrm{SD}$ ) of the theta/delta ratio was automatically set to separate SWS/NREM and REM sleep states. LFP and position data for each sleep state were also visually inspected for accuracy.

SWR detection and modulation. SWRs were detected as previously described (Cheng and Frank, 2008; Jadhav et al., 2016). Briefly, LFPs from multiple CA1 tetrodes were filtered into the ripple band $(150-250 \mathrm{~Hz})$ and the envelope of bandpassed LFPs was determined by Hilbert transform. SWRs were initially detected when the envelope exceeded a threshold (mean $+3 \mathrm{SD}$ ) on at least one tetrode. Detection of SWRs was performed only when the animals' head speed was $<4 \mathrm{~cm} / \mathrm{s}$. SWR events were then defined as times around the initially detected events during which the envelope exceeded the mean. The duration of a SWR event was defined as the difference between its onset and offset and the amplitude was defined in terms of exceeded SDs above the mean (SWR properties summarized in Fig. $4 F$ ). For sleep analysis, only SWRs that occurred in SWS periods were included. For SWR-triggered rasters, peristimulus time histograms (PSTHs), and spectral analysis, only SWRs separated from others by at least $500 \mathrm{~ms}$ were included.

For SWR modulation analysis (Fig. 2), spikes were aligned to SWR onset resulting in SWR-aligned rasters. Cells with $<50$ spikes in the SWR-aligned rasters were excluded from these analyses. To determine the significance of SWR modulation, we created 5000 shuffled rasters by circularly shifting spikes with a random jitter around each SWR, and defined a baseline response as the mean of all shuffled responses (Jadhav et al., 2016). We then compared the response in a 0-200 ms window after SWR onset (SWR response) to the baseline. We considered a cell as SWR-modulated when the mean squared difference of its actual SWR response from the baseline was $>95 \%$ of the mean squared difference of its shuffled response from the baseline (i.e., $p<0.05$ ). SWR-modulated PFC neurons were further categorized as SWR-excited or SWR-inhibited by comparing the rate in a $0-200 \mathrm{~ms}$ window after SWR onset, with the rate in a pre-SWR background window $(-500$ to $-100 \mathrm{~ms}$ before SWR onset; pre-SWR periods). SWR modulation latency (rise or fall time; Fig. $3 B$ ) was defined as the time when the SWR-aligned PSTH first increased/ decreased 1 SD away from its mean.

Spatial maps. Two-dimensional occupancy-normalized spatial maps were constructed with $2 \mathrm{~cm}$ (W-track) or $1 \mathrm{~cm}$ (sleep box) square bins and smoothed with a 2D Gaussian $(\sigma=8 \mathrm{~cm}$ for W-track; $\sigma=2 \mathrm{~cm}$ for sleep box), except that no smoothing was performed for nesting position analysis (Fig. 2I). Data from SWR periods (see SWR detection and modulation) were excluded from spatial map analysis. To measure the spatial correlation of a cell pair, the $2 \mathrm{D}$ position data were converted to linear positions measured as the distance along the track from the reward well on the center arm, which was further classified as belonging to one of four possible trajectories (i.e., center to left arm, left arm to center, center to right arm, right arm to center; Fig. 1A). Spatial correlation of a cell pair was defined as the Pearson's correlation coefficient between their linearized spatial maps.

Spatial and behavior coding properties. The sparsity of spatial firing distribution was measured using the linearized spatial maps (see Spatial maps) as the relative proportion of the map $>25 \%$ of its peak rate. To analyze the immobility coding during $\mathrm{W}$-track behavior sessions, we compared the firing rates during pre-SWR periods and high-speed moving periods as previously described (Fig. $2 H$; Jadhav et al., 2016). The pre-SWR firing rate was defined as the firing rate in a -500 to $-100 \mathrm{~ms}$ time window before SWR onset, and the high-speed firing rate was defined as the firing rate when the animals' head speed was $>10 \mathrm{~cm} / \mathrm{s}$. The firing rate bias was then defined as (pre-SWR rate - high-speed rate)/ (pre-SWR rate + high-speed rate). For nesting position coding (Fig. 2 I, J; Kay et al., 2016), we calculated the spatial firing map using the activity during waking periods in the sleep box (see Spatial maps). During these waking periods, the total number of spikes and total time spent at positions $<5 \mathrm{~cm}$ from the animals' head position at the beginning of the sleep periods (nesting positions) were defined as Nest IN, whereas Nest OUT was defined as $>5 \mathrm{~cm}$. Only periods with $>5$ min continuous sleep (see Sleep state identification) were used to define the nesting positions. Nest IN and OUT firing rates $\left(f_{\text {IN }}\right.$ and $\left.f_{\text {OUT }}\right)$ were calculated as the total number of spikes in either the Nest IN or OUT period divided by the total time of that period. The nesting position specificity index was then derived as $2 f_{\mathrm{IN}} /\left(f_{\mathrm{IN}}+f_{\text {OUT }}\right)-1$.

Theta/SWR cross-covariance and SWR co-firing. Theta periods were assigned based on a speed criterion of $>5 \mathrm{~cm} / \mathrm{s}$ and no SWRs detected on any of the CA1 tetrodes with a 3 SD criterion (Jadhav et al., 2016). Standardized cross-covariance during theta periods was computed for pairs of neurons as in previous reports (Siapas et al., 2005; Jadhav et al., 2016): cross-correlation was first computed using $10 \mathrm{~ms}$ bins, crosscovariance was then estimated by removing the expected rate of coincidence in each bin, normalized by the mean firing rates of the neurons, the bin size, and the total length of theta periods, and followed by smoothing (50 ms Gaussian window, $\sigma=16.7 \mathrm{~ms}$ ). The peak of the standardized theta cross-covariance was determined in a $\pm 200 \mathrm{~ms}$ window $\sim 0 \mathrm{~ms}$ lag. SWR cross-covariance (Fig. 3) was calculated in a similar way as theta cross-covariance, except that it was computed from the SWR-aligned rasters using a $1 \mathrm{~ms}$ bin.

SWR correlations between cell pairs (Fig. $4 A-D$ ) were measured as the cross-correlation between the spike trains of the two neurons during SWR periods using $2 \mathrm{~ms}$ bins, normalized by their mean firing rates and the bin size, and smoothed ( $11 \mathrm{~ms}$ Gaussian window, $\sigma=2 \mathrm{~ms}$; Euston et al., 2007; Cheng and Frank, 2008). Coactivation of cell pairs during SWRs was calculated as SWR co-firing as previously reported (O'Neill et al., 2008). Specifically, we took spikes occurring during SWR events (see SWR detection and modulation), divided them into $50 \mathrm{~ms}$ bins, and calculated the Pearson's correlation coefficient of the spike trains. Cells that did not respond (no spikes) during SWRs were excluded from these analyses.

Replay decoding. Replay decoding was implemented as previously described (Fig. 5A-C; Karlsson and Frank, 2009). Candidate events were defined as SWR events during which at least 5 place cells fired. To analyze replay, each candidate event was divided into $10 \mathrm{~ms}$ bins and a simple Bayesian decoder was used to calculated the probability of animals' position given the observed spikes (the posterior probability matrix): $P(X \mid$ spikes $)=P($ spikes $\mid X) P(X) / P($ spikes $)$, where $X$ is the set of all locations in the environment (spatial bin $=2 \mathrm{~cm}$ ) and we assumed a uniform prior probability of $X$. To determine $P($ spikes $\mid X)$, we assumed the firing rates of all $N$ cells are independent and Poisson distributed:

$$
P(\text { spikes } \mid X)=\prod_{i=1}^{N} P\left(\text { spikes }_{i} \mid X\right)=\prod_{i=1}^{N} \frac{\left(\tau f_{i}(X)\right)^{\text {spikes }_{i}} e^{-\tau f_{i}(X)}}{\text { spikes }_{i} !},
$$

where $\tau$ is the duration of the time window (i.e., $10 \mathrm{~ms}$ ), $f_{i}(X)$ is the expected firing rate of the $i$ th cell as a function of sampled locations $X$ and spikes $_{i}$ is the number of spikes of the $i$ th cell in the time window. Therefore, the posterior probability matrix can be derived as follows:

$$
P(X \mid \text { spikes })=C\left(\prod_{i=1}^{N} f_{i}(X)^{\text {spikes }_{i}}\right) e^{-\tau \sum_{i=1}^{N} f_{i}(X)},
$$

where $C$ is a normalization constant. We generated four posterior probability matrices for the four possible trajectories (Fig. 1A). The assessment of replay events for significance was implemented as previously 
A

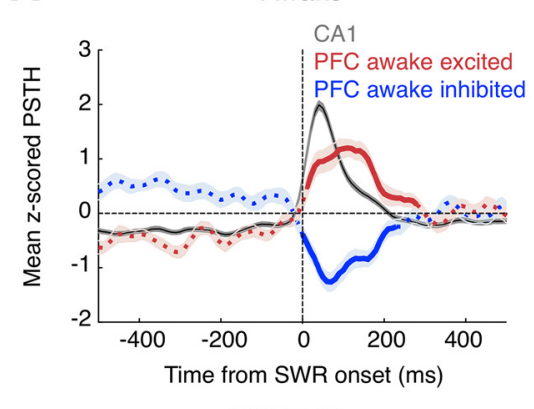

POST-Sleep

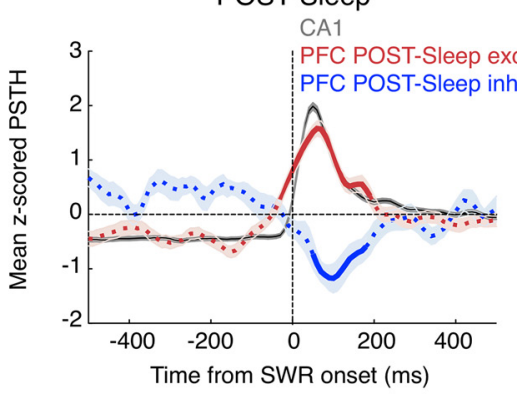

B
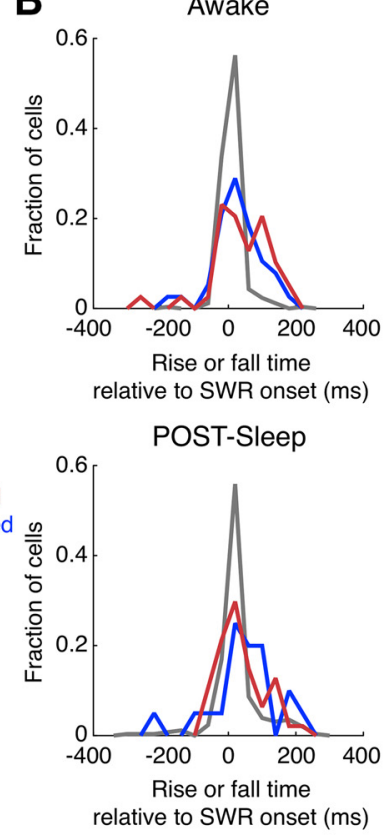

C
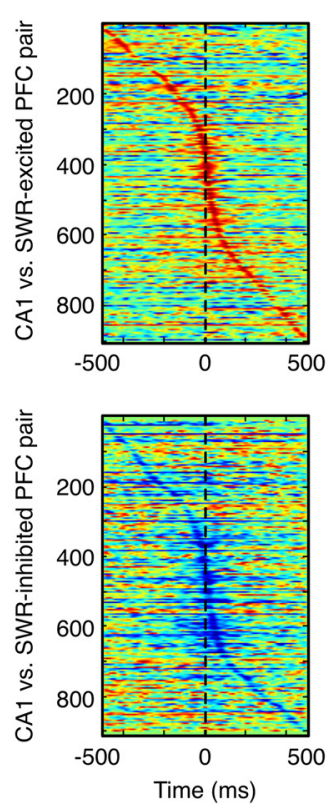

POST-Sleep
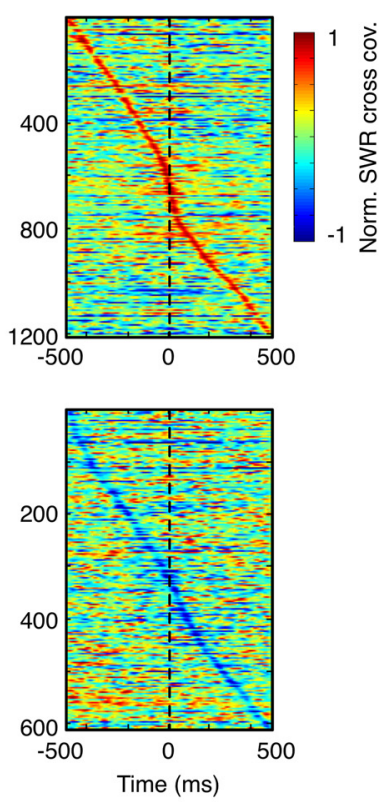

D CA1 vs. SWR-excited PFC pairs

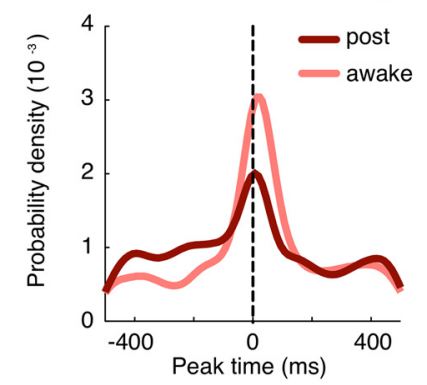

CA1 vs. SWR-inhibited PFC pairs

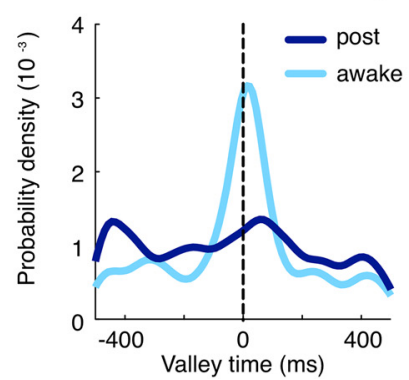

E

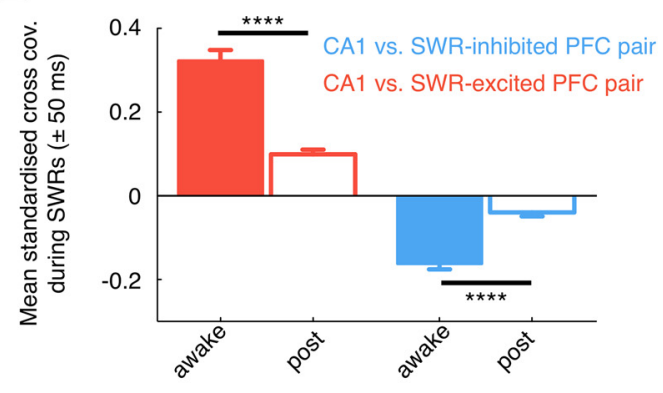

Figure 3. Stronger CA1-PFC coordination during awake SWRs than sleep SWRs. A, Population-averaged SWR-triggered PSTHs for CA1, PFC SWR-excited, and PFC SWR-inhibited cells during wakefulness (top) and post-task sleep (bottom). Shadings, SEM. Dashed PSTH curves, responses do not significantly differ from the baseline (i.e., mean firing rate during -500 to $-100 \mathrm{~ms}$ before SWR onset; $p>0.05$, Friedman test with Dunn's post hoc). B, Distributions of rise/fall time of SWR modulation for CA1, PFC SWR-excited, and PFC SWR-inhibited cells during awake SWRs (top) and post-task sleep SWRs (bottom). The latency of awake-SWR-excited PFC population occurred later than that of CA1 population $(p=0.0018)$, whereas the distributions for sleep-SWR-excited PFC and CA1 populations were similar ( $p=0.45$; rank sum tests). C, Sorted cross-covariances between CA1 and PFC cell pairs during awake SWRs (left two panels) and post-task sleep SWRs (right two panels). Top, CA1 versus SWR-excited PFC pairs (902 and 1207 pairs for awake and post-task sleep SWRs, respectively), and (bottom) CA1 versus SWR-inhibited PFC pairs (908 and 609 pairs for awake and post-task sleep SWRs, respectively). Each row shows the cross-covariance between a single pair of cells, scaled so that the peak and valley range from -1 to 1 . Pairs are sorted by the temporal lag of their peak/valley cross-covariances (i.e., peak/valley time) for CA1 versus SWR-excited PFC and CA1 versus SWR-inhibited PFC pairs, respectively. Note that there are more cell pairs with a peak/valley around time $=0$ during awake SWRs than sleep SWRs (within a $\pm 50 \mathrm{~ms}$ window at $0 \mathrm{~ms} \mathrm{lag,} \mathrm{283/902} \mathrm{and} \mathrm{253/1207} \mathrm{CA1} \mathrm{vs} \mathrm{SWR-excited} \mathrm{PFC} \mathrm{pairs} \mathrm{for} \mathrm{awake} \mathrm{and} \mathrm{post-task} \mathrm{sleep} \mathrm{SWRs:}$ $Z=5.43$ and $p=6 \mathrm{e}-8, z$ test for proportions; $300 / 908$ and 74/609 (A1 vs SWR-inhibited PF( pairs for awake and post-task sleep SWRs: $Z=9.25, p=1 \mathrm{e}-64, z$ test for proportions). D, Peak and valley time distributions for all CA1 versus SWR-excited PFC pairs (left) and CA1 versus SWR-inhibited PFC pairs (right). The distributions are estimated using kernel density (Gaussian kernel, $\sigma=$ $40 \mathrm{~ms}$ ). Note that, during awake SWRs, the distributions of both pair types are tightly concentrated $\sim 0 \mathrm{~ms}$ lag, indicating stronger synchronization between CA1 and PFC responses (kurtosis ${ }_{\text {awake, excited }}=$ -0.33 , kurtosis POST, excited $_{1}=-0.86$; kurtosis $_{\text {awake, inhibited }}=-0.28$, kurtosis Post, inhibited $=-1.11$; kurtosis is defined to be 0 for Gaussian distributions; comparing awake vs post-task sleep SWRs, $p$ values $<1 \mathrm{e}-10$, permutation tests, $n=1000$ times). $E$, Mean cross-covariances during SWRs for all cell pairs of each type. Mean cross-covariance is calculated within a $\pm 50 \mathrm{~ms}$ window at $0 \mathrm{~ms}$ lag. ${ }^{* * *} p<1 e-10$, rank sum test.

described (Karlsson and Frank, 2009). We drew 10,000 random samples from each posterior probability matrix for each decoded bin and assigned the sampled locations to that bin. Then, we performed a linear regression on the bin number versus the location points. The resulting $R^{2}$ value was then compared with 10,000 regressions in which the order of the temporal bins was shuffled. A candidate event with $p<0.05$ versus its shuffled data was considered as a replay event. The decoded trajectory was determined as the one (among the four possible trajectories) with the lowest $p$ value determined by the shuffling procedure.

Ensemble reactivation analysis. For visualization of ensemble reactivation, we calculated reactivation strength as described previously (Peyrache et al., 2009, 2010; Fig. 5D-F). Particularly, for crossregional CA1-PFC analysis, the spike trains of simultaneously recorded $N C A 1$ place cells and M PFC cells during active behavior (theta periods) were divided into $100 \mathrm{~ms}$ bins and $Z$-scored, resulted in $Q_{\text {template }}^{\mathrm{CAl}}$ and $Q_{\text {template. }}^{\mathrm{PFC}}$ A $(N \times M)$ correlation matrix $(C)$ of population activity was calculated with each element $\left(C_{i j}\right)$ representing the correlation of a CA1-

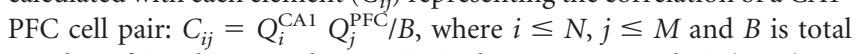
number of time bins. Further, a principal component analysis (PCA) was applied to matrix $C$ such that $C=\sum_{l} \lambda_{l} P^{(l)}$, where $P^{(l)}(N \times M)$ is the outer product of the $l$ th eigenvectors associated to an eigenvalue $\lambda_{l}$. To calculate the reactivation strength during SWRs, matrices $Q^{\mathrm{CAl}}$ and $Q^{\mathrm{PFC}}$ were constructed in the same way as $Q_{\text {template }}$, but using the spike trains during SWR events. The reactivation strength based on the first principal component was computed as follows:

$$
R_{1}(t)=\sum_{(i \leq N, j \leq M)} Q_{i t}^{C A 1} P_{i j}^{1} Q_{j t}^{P F C},
$$


A
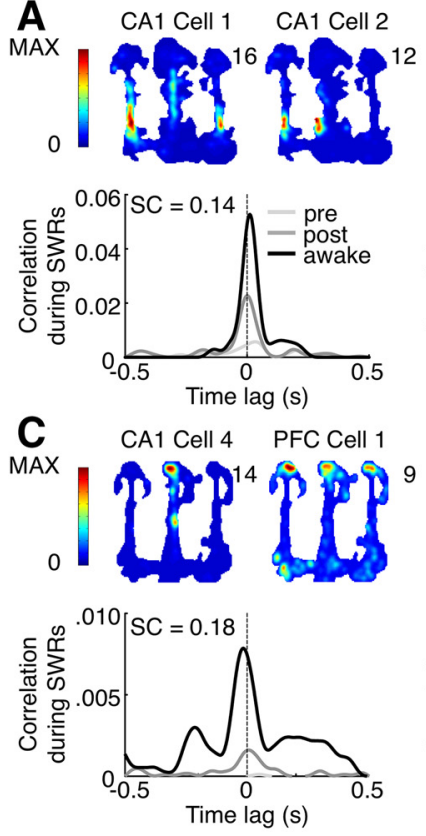

H CA1-CA1 I

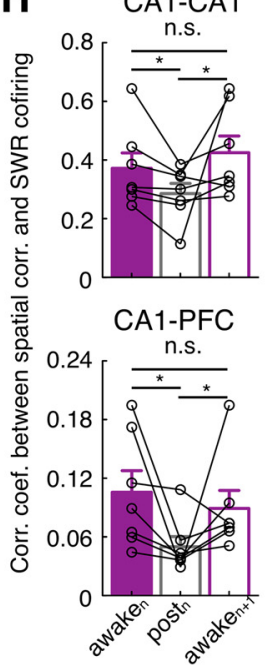

(1)
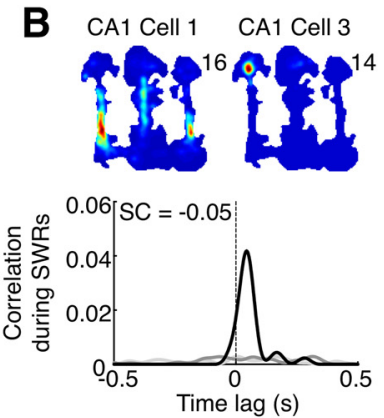

D CA1 Cell 5 PFC Cell 1
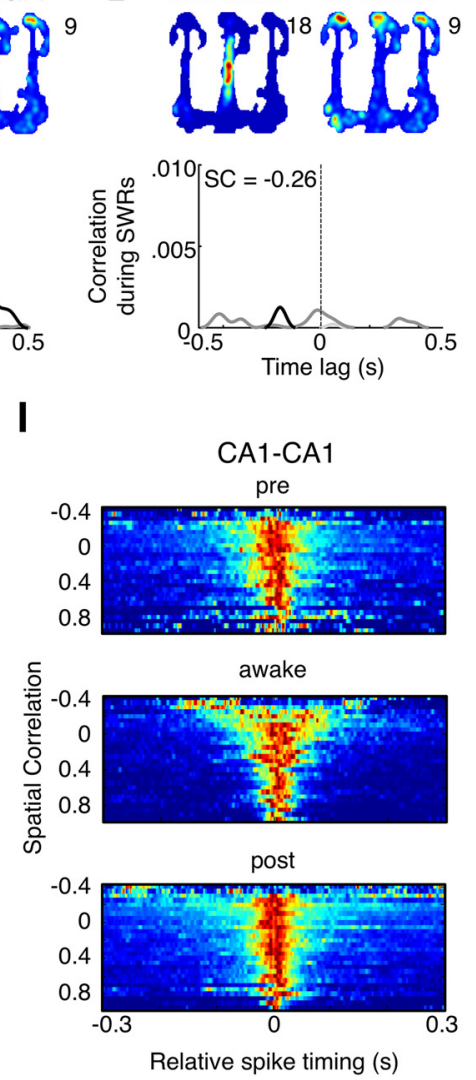
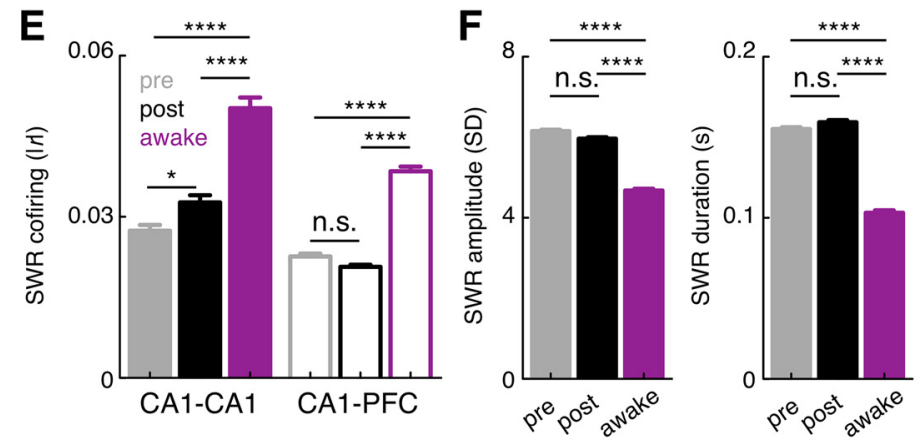

G

CA1-CA1 CA1-PFC
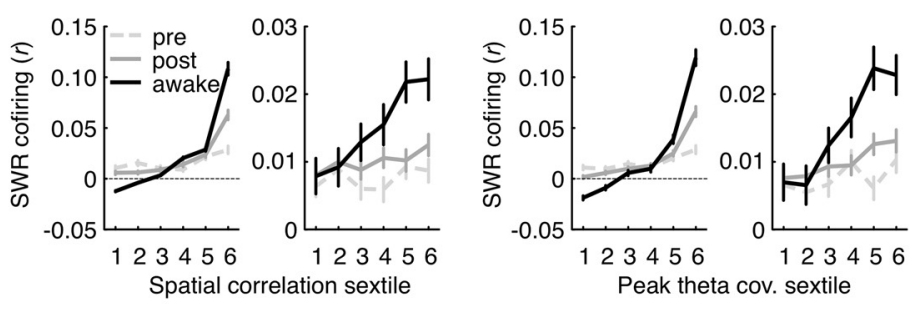

J
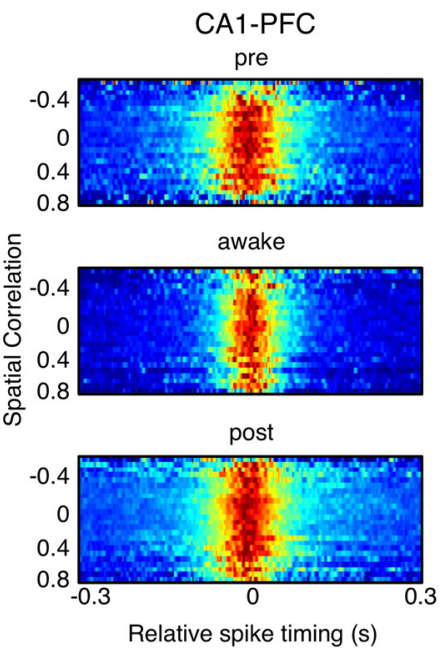

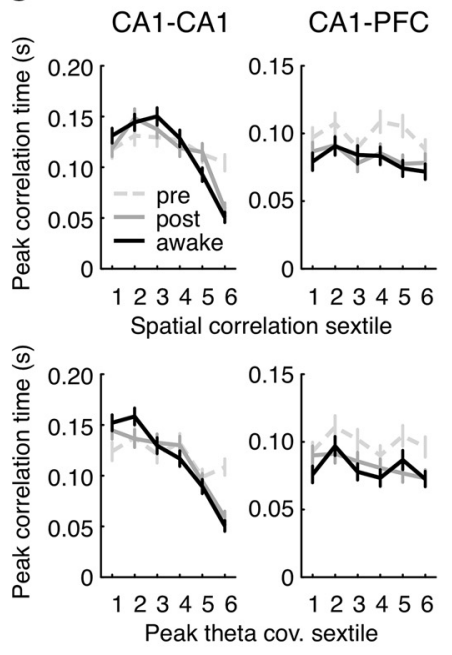

Figure 4. More structured pairwise reactivation during awake SWRs than sleep SWRs. A-D, Examples of pairwise reactivation during awake and sleep SWRs. Top, Spatial firing maps for cells during run sessions; numbers on top right of each plot denote peak spatial firing rate (MAX). Bottom, Cross-correlations for the cell pairs during SWRs; numbers on top left of each plot denote the spatial correlation (SC) of spatial firing maps. $\boldsymbol{A}$, An example CA1-CA1 pair with high spatial correlation; $\boldsymbol{B}$, an example CA1-CA1 pair with low spatial correlation; $\boldsymbol{C}$, an example CA1-PFC pair with high spatial correlation; $\boldsymbol{D}$, an example CA1-PFC pair with low spatial correlation. E, SWR co-firing of both CA1-CA1 and CA1-PFC pairs was higher during awake SWRs than sleep SWRs. n..., $p>0.05,{ }^{*} p<$ $0.05,{ }^{* * * *} p<0.0001$, rank sum test. $F$, SWR amplitude (left) and duration (right). Note that SWRs were of shorter duration and lower amplitude during wakefulness than sleep (n.S., $p>0.05$, ${ }^{* * * *} p<0.0001$, rank sum test). G, SWR co-firing as a function of spatial correlation (left two panels) and peak theta cross-covariance (right two panels). Spatial correlations and peak theta covariances are divided into six subgroups with equal number of cell pairs (sextiles; total 1762 CA1-CA1 and 1897 CA1-PFC pairs). Both CA1-CA1 and CA1-PFC pairs demonstrate stronger spatial reactivation during awake SWRs than sleep SWRs ( $Z=5.34$ and $p=9 \mathrm{e}-8, Z=2.31$ and $p=0.02$ for CA1-CA1 and CA1-PFC pairs, respectively, comparing correlation coefficients between SWR co-firing and spatial correlation in awake vs post-task sleep states). $\boldsymbol{H}$, The stronger reactivation observed during awake SWRs compared with sleep SWRs is not simply due to the order of awake and sleep sessions. Data are from two single-day animals. To account for the possibility that sleep reactivation can decay with time, we assessed the correlation between spatial correlation and SWR co-firing in three consecutive sessions (W-track, post-task sleep, and W-track). Because each animal ran eight W-track sessions (Fig. 10, seven total exploration-sleep-exploration sequences were analyzed (shown as open circles and connecting lines). Note the stronger correlation for both CA1-CA1 pairs (top) and CA1-PFC pairs (bottom) during awake SWRs than sleep SWRs (n.S., $p>0.05$, ${ }^{*} p=0.0207$; Friedman test with Dunn's post hoc for both CA1-CA1 and CA1-PFC comparisons).I, SWR reactivation for all cell pairs indicating timing relationships during CA1-CA1 reactivation. Each plot shows rows representing the normalized cross-correlations of all cell pairs against their spatial correlations. The cross-correlations are averaged across pairs that have the same spatial correlation. Left, CA1-CA1 pairs; Right, CA1-PFC pairs. J, Peak SWR-correlation time as a function of spatial correlation (top) and peak theta cross-covariance (bottom). Only CA1-CA1 reactivation demonstrates strong correlation between peak correlation time vs. spatial corr. (for CA1-CA1 pairs: $r=-0.31,-0.19,-0.05$ and $p=1 \mathrm{e}-39,1 \mathrm{e}-15,0.042$ for awake, post-task, and pre-task sleep SWRs, respectively; for CA1-PFC pairs: $r=-0.026,-0.05,-0.01$, and $p=0.27,0.04,0.66$ for awake, post-task, and pre-task sleep SWRs, respectively). This timing relationship is stronger in CA1 for awake SWRs than post-task sleep SWRs ( $Z=-3.65, p=0.0003$ for awake vs post-task sleep SWRs). Error bars indicate SEM.

where, $Q_{i t}^{\mathrm{CA1}}$ is the $Z$-scored spike count of the ith CA1 neuron at time point $t$ and $Q_{j t}^{\mathrm{PFC}}$ is for the $j$ th PFC neuron.

A similar measure of ensemble reactivation, equivalent to the average reactivation strength over time and principal components, was also computed as explained variance (EV; Fig. 5G; Kudrimoti et al., 1999). Similar to the reactivation strength, a correlation matrix $\left(C_{\mathrm{EXP}} ; N \times M\right.$ for CA1PFC ensembles and $N \times N$ for CA1-CA1 ensembles) of population activity during theta periods was calculated. In addition, three correlation matrices using spike trains during SWR events were, respectively, created for pre-task sleep, $\mathrm{W}$-track, and post-task sleep sessions $\left(C_{\mathrm{PRE}}, C_{\mathrm{AWAKE}}\right.$, 
A
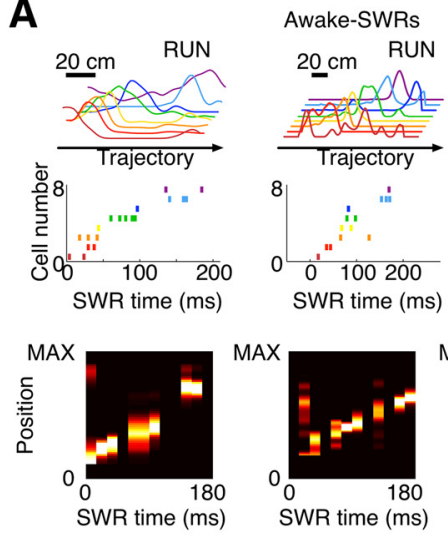

SWR time (ms)

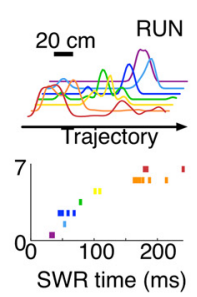

B

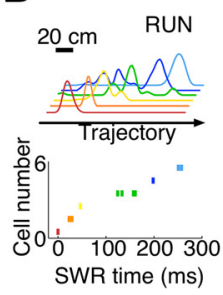

Post-task sleep-SWRs
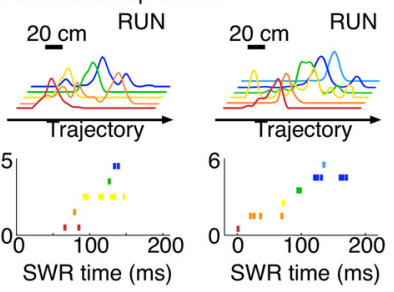

C

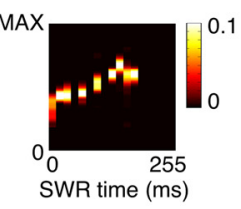

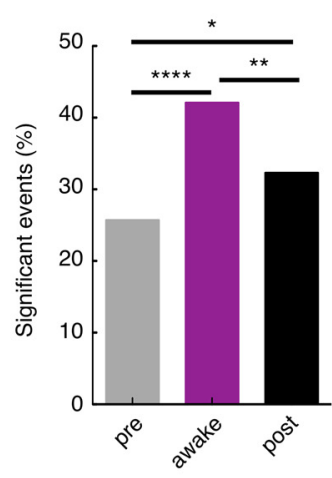

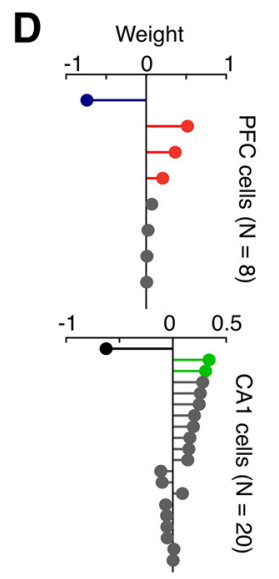

E
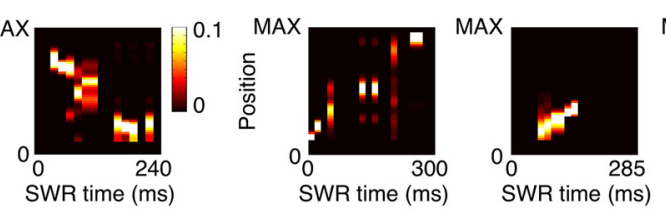

G

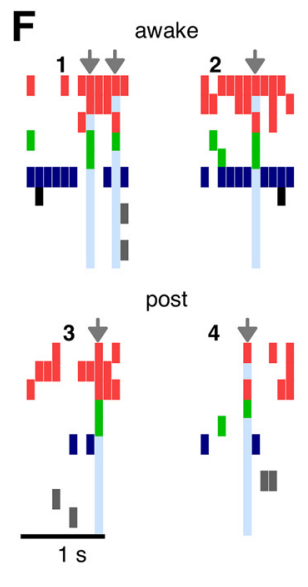

CA1-PFC

CA1-CA1
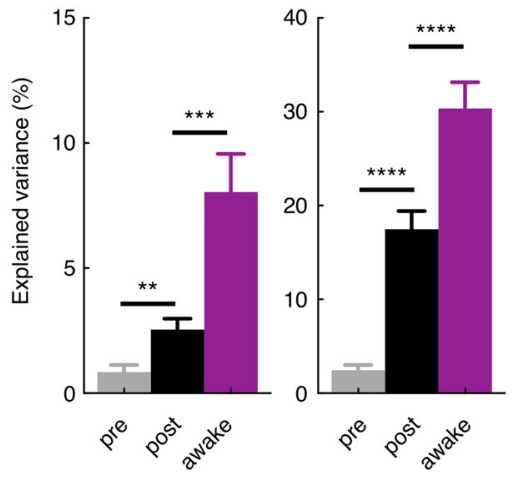

Figure 5. More structured ensemble reactivation during awake SWRs than sleep SWRs. $\boldsymbol{A}-\boldsymbol{C}$, Replay events in CA1 during awake and post-task sleep SWRs. $A, B$, Representative replay sequences of CA1 neurons during awake SWRs $(\boldsymbol{A})$ and post-task sleep SWRs $(\boldsymbol{B})$. Top, Linearized place fields of simultaneously recorded CA1 cells (color-coded) on the decoded trajectory. Middle, Sequential spiking during SWRs for the neurons shown on the corresponding top panel. Bottom, Position reconstruction for the corresponding replay events with the color scale indicating posterior probabilities of position. Note that an example reverse replay during awake SWRs is shown in $\boldsymbol{A}$ (right). C, The proportion of significant replay events in CA 1 is higher during awake SWRs than sleep SWRs ( ${ }^{*} p<$ $0.05,{ }^{* *} p<0.01,{ }^{* * *} p<0.0001 ; z$ test). $\boldsymbol{D}-\boldsymbol{F}$, Example reactivation strength of CA1-PFC ensembles during awake and sleep SWRs. $\boldsymbol{D}$, The 8 PFC and 20 CA1 neurons simultaneously recorded are ordered and color-coded to highlightneurons with high PC weights (blue, PFC cells with strong negative weights; red, PFC cells with strong positive weights; black, CA1 cells with strong negative weights; green, CA1 cells with strong positive weights). $\boldsymbol{E}$, Reactivation strength of the first signal component is greatly enhanced during awake SWRs (middle) compared with pre-task (top) and post-task (bottom) sleep SWRs. $\boldsymbol{F}$, Example synchronous events (blue shadings) of CA1-PFC ensembles (same color code as in $\boldsymbol{D}$ ) associated with the peaks shown in $\boldsymbol{E}$ (ticks: $\geq 1$ spike within $100 \mathrm{~ms}$ bins). $\boldsymbol{G}$, Explained variances of CA1-PFC (left) and CA1-CA1 (right) ensembles during awake and sleep SWRs ${ }^{* *} p<0.01,{ }^{* * *} p<0.001,{ }^{* * * *} p<0.0001$; Wilcoxon signed rank paired test). Error bars indicate SEM.

$\left.C_{\text {POST }}\right)$. These correlation matrices were further rearranged into a vector (note that because CA1-CA1 correlation matrices are symmetric, only the lower off-diagonal elements were used). To evaluate the similarity of the activity during SWRs and active movement, we calculated the Pearson's correlation coefficient between the vectors of $S W R$ correlation matrices $\left(C_{\mathrm{PRE}}, C_{\mathrm{AWAKE}}, C_{\mathrm{POST}}\right)$ and $C_{\mathrm{EXP}}$, obtaining $R_{\mathrm{EXP}, \mathrm{PRE}}, R_{\mathrm{EXP}, \mathrm{AWAKE}}$, and $R_{\mathrm{EXP}, \mathrm{POST}}$. Note that $R_{\mathrm{EXP}, \mathrm{MATCH}}$ can also be expressed as the average reactivation strength over time and principal components (Peyrache et al., 2010):

$$
R_{E X P, M A T C H}=\frac{1}{2 B^{\prime}} \sum_{i=1}^{B^{\prime}} \sum_{l} \lambda_{l} R_{l}^{M A T C H}(t)
$$

where MATCH periods could be PRE, AWAKE, or POST SWR periods, $B^{\prime}$ is the total number of time bins during MATCH periods, and $R_{l}^{\mathrm{MATCH}}(t)$ is the reactivation strength based on the $l$ th principal component at time point $t$ during MATCH periods. The explained variance of post-task sleep SWRs is further calculated as a partial correlation coefficient to subtract any pre-existing effects:

$$
E V_{P O S T}=R_{E X P, P O S T \mid P R E}^{2}=\left(\frac{R_{E X P, P O S T}-R_{E X P, P R E} R_{P R E, P O S T}}{\sqrt{1-R_{E X P, P R E}^{2}} \sqrt{1-R_{P R E, P O S T}^{2}}}\right)^{2} .
$$

Similarly, we calculated $R_{\mathrm{EXP}, \mathrm{AWAKE} \mid \mathrm{PRE}}^{2}$ as the explained variance of awake SWRs. We used the reversed-EV as the explained variance of pretask sleep SWRs:

$$
E V_{P R E}=R_{E X P, P R E \mid P O S T}^{2}
$$

Spectral analysis. All LFPs were referenced to GND for spectra analysis. Wavelet spectral analyses were used to calculate power spectra for LFPs in CA1 and PFC ( 84 levels, 1-300 Hz, Morlet wavelets; Time-Frequency Toolbox, http://tftb.nongnu.org; Fig. $6 A, B$ ), and power at each level of the wavelet transform was individually $Z$-scored. To investigate the temporal relationship between hippocampal ripples and prefrontal spindle/delta, all LFPs were first bandpass filtered (delta, 1-4 Hz; spindle, $12-18 \mathrm{~Hz}$; ripple, $150-250 \mathrm{~Hz}$ ) and the envelope was identified using the Hilbert transform. For SWR-triggered power (Fig. $6 \mathrm{C}-$ $E)$, the $Z$-scored envelopes were used and averaged across all SWRs. For cross-correlation (Fig. $6 F, G$ ), the envelopes were squared and calculated in overlapping $1 \mathrm{~s}$ window, which were log-transformed and cross-correlated as in previously reports (Siapas and Wilson, 1998; Sirota et al., 2003).

Statistical analysis. Data analysis was performed using custom routines in MATLAB (MathWorks; RRID:SCR_001622). We used nonparametric tests and two-tailed for statistical comparisons throughout the paper unless otherwise noted. To test for differences among multiple groups, we used either Kruskal-Wallis or Friedman test. Post hoc analysis was performed using Dunn's test. $p<0.05$ was considered the cutoff for statistical significance. All values reported are mean \pm SEM unless otherwise noted. 

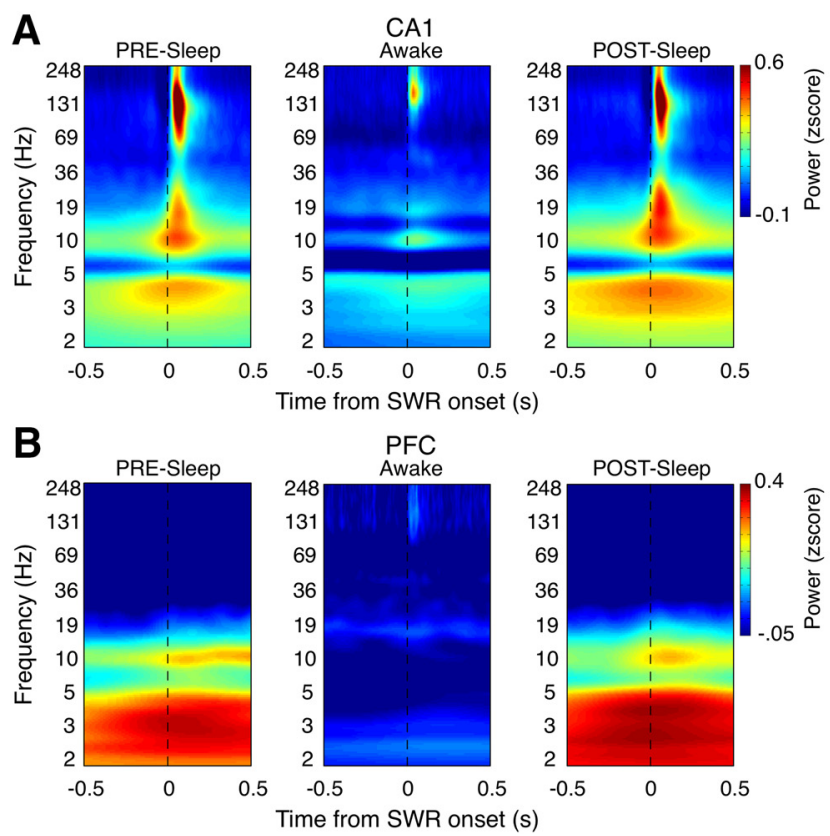

C

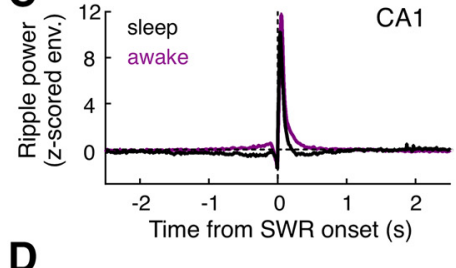

D

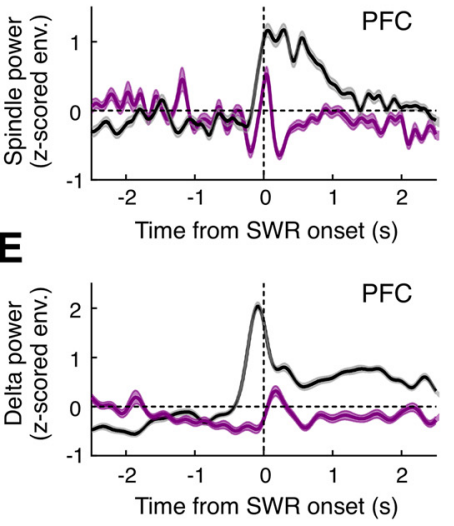

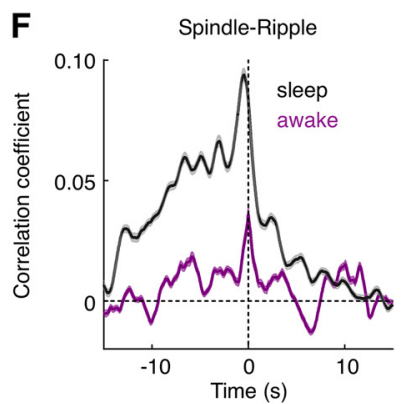

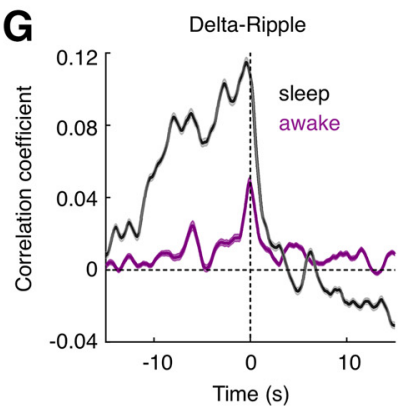

Figure 6. Network oscillations during awake and sleep SWRs. $\boldsymbol{A}, \boldsymbol{B}$, SWR-triggered average wavelet power spectrum of CA1 LFP (A) and PFC LFP (B) during pre-task sleep (left), wakefulness (middle), and post-task sleep (right). The spectrograms were Z-scored by the average power of each frequency in a given session. Note the increased delta (1-4 Hz) and spindle (12-18 $\mathrm{Hz}$ ) power in CA1 and PFC during sleep SWRs. $\boldsymbol{C}-\boldsymbol{E}$, Mean SWR-triggered envelope amplitude of ripple band $(150-250 \mathrm{~Hz})$ in $(A 1$ (C), and spindle (D) and delta (E) bands in PFC. The envelopes were derived from the Hilbert transform and Z-scored by the average amplitude in a given session. $\boldsymbol{F}, \boldsymbol{G}$, Averaged cross-correlograms of $(A 1$ ripple power versus $P F($ spindle $(\boldsymbol{F})$ and delta ( $\boldsymbol{G}$ ) power. Note that there is stronger spindle-ripple and delta-ripple coordination during SWS (or NREM). All the results are averaged across all tetrodes ( $n=138$ CA1 tetrodes and 56 PFC tetrodes) and animals ( $n=5$ rats) for all sessions. Data from pre-task and post-task sleep were combined for the sleep results shown in $\mathbf{C}-\mathbf{G}$. Shadings in $\mathbf{C}-\mathbf{G}$ indicate SEM.

\section{Results}

We used multisite, multielectrode recordings to simultaneously record the activity of neurons in dorsal CA1 region of hippocampus and medial prefrontal cortex of rats learning a W-track spatial alternation task (Fig. 1A), as previously described (Jadhav et al., 2016). The task requires animals to visit the two outer arms of the $\mathrm{W}$-track in an alternating sequence (outbound component), interleaved with visits to the center arm (inbound component). Animals learned the task in multiple run sessions in an initially novel environment, either over a period of $5 \mathrm{~d}$ (multiday/5-day learning, $n=3$ animals, 2 run sessions each day; Jadhav et al., 2016), or over the course of a single day (single-day learning, $n=$ 2 animals, 8-12 run sessions), with interleaved sleep sessions prior, between and after run sessions (see Materials and Methods; schematic of timeline in Fig. $1 B, C$; all results remained unchanged using data from just the multiday animals). We monitored activity of CA1 and PFC ensembles continuously for each day of recording over interleaved run and sleep sessions. A total of 346 CA1 neuron and 193 PFC neurons were recorded from five animals (Table 1), excluding a small number of fast-spiking, narrow-waveform cells, as well as cells with $<100$ spikes in a given session (see Materials and Methods; Jadhav et al., 2016).

PFC modulation differs for awake and sleep SWRs

Awake and sleep SWRs are both thought to reactivate behavioral experiences, but whether PFC neurons show similar or different patterns of modulation, indicative of possible differences in memory functions, is not known. We therefore first compared patterns of modulation in PFC neurons during awake and sleep SWRs. Awake SWRs were detected using movement speed and power in the ripple band $(150-250 \mathrm{~Hz})$ for CA1 tetrodes, as previously reported (Jadhav et al., 2016; see also Materials and
Table 2. Sleep parameters

\begin{tabular}{|c|c|c|c|c|c|c|c|}
\hline \multirow[b]{2}{*}{ Animal } & \multirow[b]{2}{*}{ Day } & \multicolumn{3}{|c|}{ Pre-task sleep } & \multicolumn{3}{|c|}{ Post-task sleep } \\
\hline & & $\begin{array}{l}\text { Total } \\
\text { sleep } \\
\text { time }\end{array}$ & $\begin{array}{l}\text { SWS or } \\
\text { NREM }\end{array}$ & REM & $\begin{array}{l}\text { Total } \\
\text { sleep } \\
\text { time }\end{array}$ & $\begin{array}{l}\text { SWS or } \\
\text { NREM }\end{array}$ & REM \\
\hline \multirow[t]{5}{*}{$\mathrm{HPa}$} & 1 & 28.0 & 24.4 & 3.60 & 19.2 & 18.2 & 1.00 \\
\hline & 2 & 31.7 & 28.5 & 3.22 & 26.9 & 24.8 & 2.05 \\
\hline & 3 & 30.6 & 27.8 & 2.76 & 25.3 & 23.1 & 2.06 \\
\hline & 4 & 30.6 & 30.3 & 0.20 & 19.0 & 18.5 & 0.56 \\
\hline & 5 & 21.4 & 20.7 & 0.62 & 23.1 & 22.7 & 0.33 \\
\hline \multirow[t]{5}{*}{$\mathrm{HPb}$} & 1 & 29.7 & 28.4 & 1.27 & 28.4 & 27.0 & 1.44 \\
\hline & 2 & 24.3 & 24.3 & 0 & 30.0 & 28.9 & 1.06 \\
\hline & 3 & 27.9 & 26.7 & 1.20 & 34.2 & 32.0 & 2.21 \\
\hline & 4 & 26.8 & 24.8 & 1.97 & 32.4 & 28.5 & 3.80 \\
\hline & 5 & 24.8 & 24.2 & 0.56 & 27.9 & 24.8 & 3.07 \\
\hline \multirow[t]{5}{*}{$\mathrm{HPC}$} & 1 & 27.3 & 25.5 & 1.78 & 25.8 & 22.4 & 3.47 \\
\hline & 2 & 28.7 & 26.8 & 1.93 & 24.6 & 23.1 & 1.50 \\
\hline & 3 & 25.8 & 25.4 & 0.40 & 17.3 & 17.2 & 0 \\
\hline & 4 & 27.8 & 27.0 & 0.86 & 20.1 & 18.0 & 2.09 \\
\hline & 5 & 28.0 & 27.1 & 0.84 & 20.3 & 18.0 & 2.35 \\
\hline ER1 & 1 & 8.7 & 8.2 & 0 & 35.7 & 32.4 & 0.59 \\
\hline KL8 & 1 & 28.6 & 20.0 & 8.00 & 34.1 & 27.8 & 5.16 \\
\hline Mean \pm SEM & & $26.5 \pm 1.3$ & $24.7 \pm 1.2$ & $1.7 \pm 0.5$ & $26.1 \pm 1.4$ & $24.0 \pm 1.2$ & $1.9 \pm 0.3$ \\
\hline
\end{tabular}

Summary of sleep parameters from each animal recorded on each experiment day. All measures are in minutes.

Methods). To detect sleep SWRs, we first classified REM and SWS (or NREM) stages in the sleep sessions using a criterion of thetadelta ratio during immobility, similar to other studies (Mizuseki et al., 2011; Kay et al., 2016; example of sleep stage classification in Fig. 1D; details of sleep period lengths in Table 2). Further analysis was restricted only to SWRs occurring during NREM/SWS periods (sleep sessions before behavior: Pre-task Sleep, and after behavior: Post-task Sleep; Fig. 1C).

Surprisingly, we found that PFC neurons showed different patterns of modulation during sleep SWRs compared with their 
modulation during awake SWRs (Fig. $2 A, B$; only post-task sleep is shown). We previously reported that PFC neurons show equally prevalent patterns of excitation and inhibition during awake SWRs (Jadhav et al., 2016), whereas CA1 neurons are known to be primarily positively modulated during SWR reactivation (Jadhav et al., 2016; Kay et al., 2016). Figure 2A-E illustrates the excitation and inhibition of PFC neurons seen during awake SWRs [SWR-modulated: 40\% (77/193), SWR-excited: 20\% (39/193), SWR-inhibited: 20\% (38/193); see Materials and Methods for quantification of SWR-modulation; example rasters and PSTHs in Fig. 2A; population PSTHs in Fig. 2B]. In contrast, during sleep SWRs, PFC neurons were predominantly excited [Fig. 2 B, C; Table 1; Pre-task Sleep: SWR-modulated: 29\% (57/ 193), SWR-excited: 20\% (39/193), SWR-inhibited: 9\% (18/193); Post-task Sleep: SWR-modulated: 34\% (67/193), SWR-excited: 24\% (47/193), SWR-inhibited: 10\% (20/193); $z$ tests comparing the proportions of total SWR-modulated PFC cells: $Z=2.14$ and $p=0.03, Z=1.05$ and $p=0.29$ for awake SWRs vs Pre-task and Post-task Sleep SWRs, respectively].

There were significantly fewer SWR-inhibited cells in sleep compared with the awake state $(z$ tests for the proportions of SWR-inhibited PFC cells: $Z=-2.89$ and $p=0.004, Z=-2.56$ and $p=0.01$ for Pre-task and Post-task Sleep SWRs vs awake SWRs; $z$ tests for the proportions of SWR-excited PFC cells: $Z=$ 0 and $p=1, Z=-0.98$ and $p=0.33$ for Pre-task and Post-task Sleep SWRs vs awake SWRs). Remarkably, there was no correlation between awake-SWR modulation and Post-task Sleep-SWR modulation (Fig. $2 C$; $n=193$ cells, $r=0.029, p=0.68$, Spearman correlation). Indeed, whereas awake-SWR-excited PFC neurons overall maintained net positive modulation during both pre-task and post-task sleep SWRs $(p=0.018$ and 0.011 for Pre-task and Post-task Sleep, respectively, rank sum tests for differences from $0)$, awake-SWR-inhibited neurons showed a significant bias to net positive modulation during post-task sleep $(p=0.01)$, and showed no overall bias during pre-task sleep ( $p=0.69$, rank sum tests for differences from 0; Fig. 2D,E). We thus found a lack of correlation between PFC modulation for awake versus sleep SWRs, with a strong bias toward excitation during sleep. Although the different modulation patterns of PFC neurons might be driven by global state-dependent network changes during awake versus sleep states, such as synaptic connectivity, we confirmed that this bias was not due to methodological issues, such as differences in detection of excitation versus inhibition (excitation strength: $0.095 \pm 0.010$ for awake SWRs; $0.097 \pm 0.009$ for Posttask Sleep SWRs; inhibition strength: $-0.080 \pm 0.007$ for awake SWRs; $-0.081 \pm 0.008$ for Post-task Sleep SWRs; excitation and inhibition strengths were measured as SWR modulation index of PFC neurons; $p=0.85$ and 0.90 for comparing excitation and inhibition during awake vs Post-task Sleep SWRs, respectively, rank sum tests), and differences in firing rates (Fig. 2F). Further, we also did not find any differences in basic spatial coding properties; spatial sparsity was similar for the population of awake and sleep SWR-excited and SWRinhibited neurons (Fig. 2G).

What coding properties could account for the differences in the proportions of positively and negatively modulated PFC cells across states? We had previously reported that awake SWRinhibited PFC neurons preferentially encoded periods of immobility and slow-speed movement, whereas awake SWRexcited PFC neurons preferentially encoded periods of movement (Jadhav et al., 2016). As expected, we found that awake SWR-modulated PFC neurons showed preference for immobility (SWR-inhibited PFC neurons) and movement (SWR- excited PFC neurons; $p=0.004$, rank sum test; Fig. $2 H$ ). We therefore asked whether similar coding properties explained the PFC modulation patterns during sleep. Indeed, immobility coding neurons that encode nesting position of the animal during sleep have been reported in CA1 and CA2 regions of hippocampus (Jarosiewicz et al., 2002; Kay et al., 2016). Using an identical nesting position specificity index (see Materials and Methods), we found that the small number of sleep SWRinhibited neurons in PFC preferentially encoded nesting position of the animal compared with sleep SWR-excited PFC neurons $(p=0.005$, rank sum test; Fig. $2 I, J)$. Note that these immobility coding properties explain the elevated pre-SWR firing relative to baseline rate of SWR-inhibited PFC neurons shown in Figure $2 A$ (e.g., Cells 1-2 during awake SWRs and Cell 4 during post-task sleep SWRs). Thus, the pattern of inhibition of PFC neurons was explained best by the movement state coding properties during both waking behavior and sleep, possibly contributing to differential modulation in the two cases.

\section{Coordination in CA1-PFC network during SWRs}

During awake SWR reactivation events, hippocampal-cortical interaction is manifest in the temporal coordination of CA1 and PFC spiking, which also points to exchange of information between the networks during reactivation (Jadhav et al., 2016). The differences in PFC modulation properties during awake versus sleep SWRs that we found raise the possibility that relative coordination of CA1 and PFC neurons during SWR-reactivation may also be different between the two states. We found that within each category of SWR-modulated PFC neurons (i.e., SWRexcited or SWR-inhibited), the population response profiles were similar during awake and sleep SWRs (Fig. $3 A$, top vs bottom), except that sleep-excitation of PFC neurons tended to occur earlier relative to SWR-onset (Fig. 3A, bottom). The modulation latency of neurons, measured as the time of firing rate increase/ decrease to $1 \mathrm{SD}$ above/below the mean (rise or fall time), was also significantly later for awake-SWR-excited PFC neurons than CA1 neurons ( $p=0.0018$, rank sum test), whereas the distributions of CA1 and sleep-SWR-excited PFC neurons were similar ( $p=$ 0.45 , rank sum test; Fig. $3 B$; see Materials and Methods). These average timing profiles suggest a difference in SWR-associated CA1-PFC coordination, which we tested directly using crosscovariance analysis of CA1 neurons and SWR-modulated PFC neurons. Figure $3 C$ shows the normalized cross-covariance during awake and post-task sleep SWRs between pairs of CA1 versus SWR-excited PFC neurons, and CA1 versus SWR-inhibited PFC neurons, respectively. For both SWR-excited and SWR-inhibited PFC neurons, CA1-PFC activity showed a stronger clustering of peaks (or troughs for SWR-inhibited neurons) in cross-covariance at $0 \mathrm{~ms}$ lag for awake SWRs compared with post-task sleep SWRs (Fig. 3C,D), indicating stronger synchronization and engagement of activity during awake SWRs. This stronger coordination is also apparent in significant differences in mean cross-covariance in a \pm $50 \mathrm{~ms}$ window at $0 \mathrm{~ms}$ lag for awake-modulated versus sleepmodulated neurons (Fig. $3 E ; p=5 \mathrm{e}-11$ for the pairs of CA1 vs SWR-excited PFC neurons, $p=5 e-21$ for the pairs of CA1 vs SWR-inhibited PFC neurons, rank sum tests). Thus, in addition to the differences in single-neuron modulation properties in $\mathrm{PFC}$, we found stronger coordination in pairwise measures of CA1-PFC activity during awake SWRs.

\section{Stronger pairwise spatial reactivation during awake SWRs}

Reactivation of neural patterns representing behavioral experience is thought to be important for memory (Battaglia et al., 
2011; Carr et al., 2011; Buzsáki, 2015; Roumis and Frank, 2015). The differences in modulation and coordination in the CA1-PFC network during awake versus sleep SWRs led us to ask whether reactivation was also different, by directly comparing reactivation during these two states. We first used a pairwise measure, which assesses the relationship between spatial correlation and SWR co-firing to quantify both CA1-CA1 and CA1-PFC spatial reactivation (Fig. 4). This method is similar to those used previously to quantify pairwise CA1-CA1 reactivation (O'Neill et al., 2008) and CA1-PFC reactivation (Jadhav et al., 2016), and tests whether there is a relationship between the degree of spatial correlation (or place field similarity) between pairs of neurons and their co-firing during SWRs (see Materials and Methods). Spatial reactivation in CA1-CA1 pairs has been shown to increase in post-task sleep SWRs relative to pre-task sleep SWRs (O'Neill et al., 2008), and spatial reactivation is also seen in CA1-PFC pairs during awake SWRs (Jadhav et al., 2016). This pairwise measure quantifies SWR replay of waking experience, thought to be critical for memory processes (see Discussion).

Examples of pairs of CA1-CA1 and CA1-PFC neurons with high and low spatial correlations are shown in Figure $4 A-D$, along with the associated cross-correlations during SWRs (during awake, post-task sleep, and pre-task sleep SWRs). As seen in these examples, the coactivation among CA1-CA1 and CA1-PFC cell pairs during awake SWRs was higher than that during sleep SWRs (Fig. 4E), consistent with the results in Figure 3. The stronger coordination during awake SWRs occurred despite shorter durations and lower amplitudes of awake SWRs compared with sleep SWRs (Fig. 4F). The effect persisted while controlling for the durations of SWRs using subsampling (data not shown), confirming stronger temporal coordination of spiking during awakeSWR reactivation.

Moreover, cell pairs with higher spatial correlation tended to have higher SWR correlation (Fig. $4 A$ vs $B, C$ vs $D$ ). Overall, in the population, we found that the relationship between spatial correlation and SWR co-firing was stronger for awake SWRs than sleep SWRs for both CA1-CA1 pairs (Fig. $4 G$, far left; $n=1762$ pairs; $r=0.43,0.30,0.08$, and $p=1 \mathrm{e}-78,1 \mathrm{e}-79,0.013$, for awake, post-task, and pre-task sleep SWRs, respectively; $Z=$ 4.34, $p=8 \mathrm{e}-5$ for awake vs post-task sleep SWRs), and CA1PFC pairs (Fig. 4G, middle left; $n=1897$ pairs; $r=0.19,0.05$, 0.01 , and $p=2 \mathrm{e}-7,0.046,0.69$ for awake, post-task, and pre-task sleep SWRs, respectively; $Z=2.23, p=0.026$ for awake vs posttask sleep SWRs), suggesting stronger spatial reactivation during awake compared with sleep SWRs. We also observed more structured awake reactivation compared with sleep reactivation in both subsequent and preceding rest box sessions for the singleday animals (Fig. 4H), indicating that the observed differences are not simply because sleep reactivation degraded with time (Kudrimoti et al., 1999; Eschenko et al., 2008). Further, we saw similar results using another measure of behavioral relationships instead of spatial correlations, namely theta cross-covariance, which quantifies behavioral correlations between pairs of neurons during hippocampal theta oscillations that predominate during exploratory behavior (Fig. $4 G$, middle and far right; $r=$ $0.50,0.36,0.07$, and $p=1 \mathrm{e}-113,1 \mathrm{e}-114,0.002$ for CA1-CA1 pairs, and $r=0.14,0.07,0.02$, and $p=2 \mathrm{e}-10,0.002,0.30$ for CA1-PFC pairs during awake, post-task, and pre-task sleep SWRs, respectively; $Z=5.34$ and $p=9 \mathrm{e}-8, Z=2.31$, and $p=0.02$ for CA1-CA1 and CA1-PFC pairs, respectively, comparing awake vs post-task sleep SWRs).

We also examined whether timing relationships were preserved during SWR reactivation. For CA1-CA1 pairs, it has been shown that there is a correlation between the distance between place-field peaks (a measure of spatial correlation) and relative spike timing during SWR reactivation, and this correlation is stronger during awake periods than post-task quiescence (Karlsson and Frank, 2009). Figure $4 I$ shows the relationship between spatial correlation and relative spike timing for all CA1-CA1 and CA1PFC pairs during awake, post-task, and pre-task sleep SWRs. The reactivation of CA1-CA1 pairs exhibited an expanding $\mathrm{V}$ shape especially during awake SWRs, indicating a timing relationship. In contrast, the pattern of CA1-PFC pairwise reactivation showed a tight concentration at $0 \mathrm{~ms}$ lag. To verify the timing relationship, we measured the correlation between the degree of spatial correlation and the peak time of SWR correlation. For CA1-CA1 pairs, awake SWRs showed stronger correlation than post-task sleep SWRs (Fig. 4J, top left; $n=1762$ pairs; $r=-0.31,-0.19$, -0.05 , and $p=1 \mathrm{e}-39,1 \mathrm{e}-15,0.042$ for awake, post-task, and pre-task sleep SWRs, respectively; $Z=3.65, p=0.0003$ for comparing correlation coefficients of awake vs post-task sleep SWRs). We however did not find strong timing reactivation for CA1-PFC pairs (Fig. 4J, top right; $n=1897$ pairs; $r=-0.026,-0.05$, -0.01 , and $p=0.27,0.04,0.66$ for awake, post-task, and pre-task sleep SWRs, respectively; $Z=7.32, p=1 \mathrm{e}-12$ for comparing correlation coefficients of CA1-CA1 vs CA1-PFC pairs during post-task sleep SWRs). This lack of timing reactivation in CA1-PFC pairs suggests that although there is coordinated reactivation in the CA1-PFC network, this is unlikely to manifest as reactivation of sequences across regions, but rather as synchronization of cell assemblies (Peyrache et al., 2009; van de Ven et al., 2016).

\section{Stronger ensemble reactivation during awake SWRs}

Ensemble measures of reactivation have been linked to memory (Peyrache et al., 2009; van de Ven et al., 2016), so we next asked whether the stronger reactivation seen in pairwise measures was also seen in measures of ensemble reactivation, both within CA1 and in the CA1-PFC network. Replay of CA1 sequences was quantified as described previously (Karlsson and Frank, 2009; see Materials and Methods). Examples of CA1 replay sequences, both in the forward and backward directions, during awake and sleep SWRs are shown in Figure 5, $A$ and $B$. We found that there was a significantly higher fraction of replay events during awake SWRs than post-task sleep SWRs (Fig. 5C; $n=167 / 397,147 / 455$, $116 / 451$ significant replay events of candidate events during awake, post-task, and pre-task sleep SWRs; $Z=2.95$ and $p=$ 0.0032 for awake vs post-task sleep SWRs, and $Z=2.18$ and $p=$ 0.029 for post-task vs pre-task sleep SWRs), which has been suggested in previous studies (Karlsson and Frank, 2009; Grosmark and Buzsáki, 2016). Note that because pre-task sleep sessions did not necessarily precede the first novel exposure of the environment (Fig. 1C), the replay events in the pre-task sleep sessions are not the same as hypothesized preplay events (Dragoi and Tonegawa, 2011; Ólafsdóttir et al., 2015).

To examine ensemble reactivation in the CA1-PFC network, we used a measure of synchronization during reactivation of behavioral experiences, namely reactivation strength and explained variance (Kudrimoti et al., 1999; Peyrache et al., 2009, 2010). An example of computation of reactivation strength of CA1-PFC ensemble is shown in Figure $5 D-F$. Briefly, during W-track waking behavior, PCA was applied to the cross-correlation matrix of simultaneously recorded CA1 and PFC spike trains binned at 100 $\mathrm{ms}$ as previously described (Peyrache et al., 2009). The principal components (PCs) described the contribution of each neuron to the identified ensemble ( $\mathrm{PC}$ weight; Fig. 5D). At a given bin of 
SWR time, reactivation strength assesses the similarity between the identified coactivation patterns during waking behavior and the SWR period neural activity (reactivation of signal component in Fig. 5D is shown in E; see Materials and Methods). These peaks of reactivation strength correspond to synchronous spiking events of principal CA1 and PFC neurons during SWRs (Fig. 5F). Notably, these synchronous events reappeared more frequently and strongly during awake SWRs than sleep SWRs (Fig. 5E). To quantify the difference in synchrony during awake and sleep SWR reactivation, we further computed the average reactivation strength (Peyrache et al., 2010) or EV (Kudrimoti et al., 1999). We found that for both CA1 and CA1-PFC ensembles, awake SWRs had significantly higher values of explained variance than post-task sleep SWRs (Fig. 5G; $n=34$ run sessions and 17 pretask/post-task sleep sessions; $p=8 \mathrm{e}-5$ and 0.0001 for CA1 ensembles and CA1-PFC ensembles, respectively, comparing awake vs post-task sleep; $p=7 e-7$ and 0.0026 for CA1 ensembles and CA1-PFC ensembles, respectively, comparing pre-task vs posttask sleep; Wilcoxon signed rank paired tests). Note that these measures focus on synchronized activity in CA1-PFC ensembles, using a time-scale equivalent to the bin size (similar results were obtained using a bin size of $50 \mathrm{~ms}$ ). We therefore found that even at an ensemble level, reactivation was stronger during awake SWRs than sleep SWRs.

\section{Network oscillations during awake and sleep SWRs}

Coordination of cortical delta $(1-4 \mathrm{~Hz})$ and spindle $(12-18 \mathrm{~Hz})$ oscillations with SWRs is a prominent feature of sleep reactivation (Siapas and Wilson, 1998; Sirota et al., 2003; Peyrache et al., 2011; Maingret et al., 2016; Miyawaki and Diba, 2016; Rothschild et al., 2017), and this coordination is thought to be important for memory consolidation (Sejnowski and Destexhe, 2000; Inostroza and Born, 2013; Maingret et al., 2016). Because we observed stronger reactivation during awake SWRs, we next asked whether there are any differences in coordination of network oscillations during awake versus sleep SWRs. Indeed, we found increases in spindle and delta power during sleep SWRs (see Materials and Methods for details of spectral analysis). Figure $6, A$ and $B$, shows the averaged SWR-aligned spectrograms in CA1 and PFC, respectively ( $n=138$ CA1 tetrodes and 56 PFC tetrodes in 34 sessions for awake, 17 sessions for pre-task/post-task sleep). Unlike sleep SWRs, awake SWRs did not show a corresponding increase in cortical spindle and delta band power (Fig. 6B). We further examined the average cortical power in different frequency bands around SWRs (using envelope amplitude in a given frequency band derived from the Hilbert transform; see Materials and Methods), and found significant differences in cortical spindle and delta power during awake versus sleep SWRs (Fig. 6D,E; $p=1 \mathrm{e}-14$ and $1 \mathrm{e}-25$ for spindle and delta power in a $\pm 1 \mathrm{~s}$ window around awake vs sleep SWRs, rank sum tests). To investigate the coordination between spindle/delta and ripples, we calculated their cross-correlations (Siapas and Wilson, 1998; Sirota et al., 2003; see Materials and Methods). We found the spindleripple coordination and the delta-ripple coordination during SWS (or NREM) was significantly enhanced compared with wakefulness (Fig. 6F, G; $p=1 \mathrm{e}-76$ and $1 \mathrm{e}-94$ for spindle-ripple and delta-ripple correlation coefficients in a $\pm 1 \mathrm{~s}$ window at $0 \mathrm{~ms}$ lag, rank sum tests).

Thus, coordination between hippocampal SWRs and cortical delta and spindle oscillations that is seen during sleep was not observed during awake SWRs, even though we found that reactivation was stronger during awake SWRs (Figs. 3-5). These results therefore indicate that coordination of hippocampal- cortical network oscillations, a prominent feature of sleep reactivation, is not required for coordinated reactivation seen during awake SWRs.

\section{CA1-PFC spatial reactivation is strongest during initial learning}

Finally, given the proposed role of reactivation in learning (Singer et al., 2013; Yu and Frank, 2015; Papale et al., 2016), we asked how reactivation during awake and sleep SWRs changes over the course of learning the task on the novel W-track. Previous studies have reported increased CA1 co-firing during awake SWRs in a novel environment (Cheng and Frank, 2008), but how CA1-PFC reactivation changes over learning of a spatial task during awake and sleep SWRs has not been investigated.

Because our data consisted of learning on two different time scales (Fig. 1C; 3 animals learned over the course of $5 \mathrm{~d}$, and 2 animals learned in a single day), we first examined how reactivation changed in the multiday learning group. Learning curves for all three animals for both the inbound and outbound components are shown in Figure $7 \mathrm{~A}$. We asked how spatial reactivation, quantified using pairwise and ensemble measures (Figs. 4, 5) changed over learning. Figure $7 B$ shows awake pairwise reactivation, quantified as the relationship between awake SWR co-firing versus spatial reactivation for both CA1-CA1 and CA1-PFC pairs, over successive days of learning. Note the decrease in reactivation for awake SWRs, especially in CA1-PFC, starting with high reactivation (high correlation between SWR co-firing and spatial correlation) on the first (novel exploration) day when the animal begins to learn the task via trial-and-error, to lower reactivation on the latter days when performance starts to stabilize (days 4-5 are combined for a better comparison of data). Although SWR rate tended to decrease with decrease in novelty, as expected (Foster and Wilson, 2006; Cheng and Frank, 2008), overall spatial correlation and SWR co-firing remained relatively stable in the CA1-CA1 and CA1-PFC populations (Fig. 7C-F). Thus, the decrease in CA1-PFC reactivation was not due a decrease in overall co-firing, but rather reflects a decrease in relationship between spatial correlation during behavior and SWR co-firing.

Significant within-CA1 reactivation was observed in latter days for both awake and sleep SWRs, also apparent in other measures: theta covariance versus SWR co-firing, and the ensemble measure of explained variance (Fig. $7 G$; for days $4-5, p$ values $=$ $1 \mathrm{e}-25$ for correlation coefficients between SWR co-firing vs spatial correlation and SWR co-firing vs theta peak covariance). As previously reported (O’Neill et al., 2008), we did see a decrease in CA1 co-firing change from pre-task to post-task sleep over repeated explorations (Fig. $7 F$ ). In contrast, CA1-PFC reactivation declined sharply and was indistinguishable from chance level on latter days (Fig. $7 H$; For days $4-5, p=0.58$ and 0.52 for correlation coefficients between SWR co-firing vs spatial correlation and theta peak covariance, respectively; see Materials and Methods). The difference between awake and sleep SWRs was most prominent during the initial day of novelty (spatial correlation vs awake SWR co-firing, $Z=3.82$ and $p=0.0001$ for day 1 vs days $4-5, z$ test; explained variance, $p=1 \mathrm{e}-44$ for day 1 vs days 4 and $5, p$ value is from bootstrapping, $n=500$ times). This also corresponds to a period of early learning in the task, with awake CA1PFC reactivation especially enhanced in this period.

These results thus suggest that awake reactivation is prominent in the hippocampal-prefrontal network during initial learning in novel environments. To further investigate the specific 

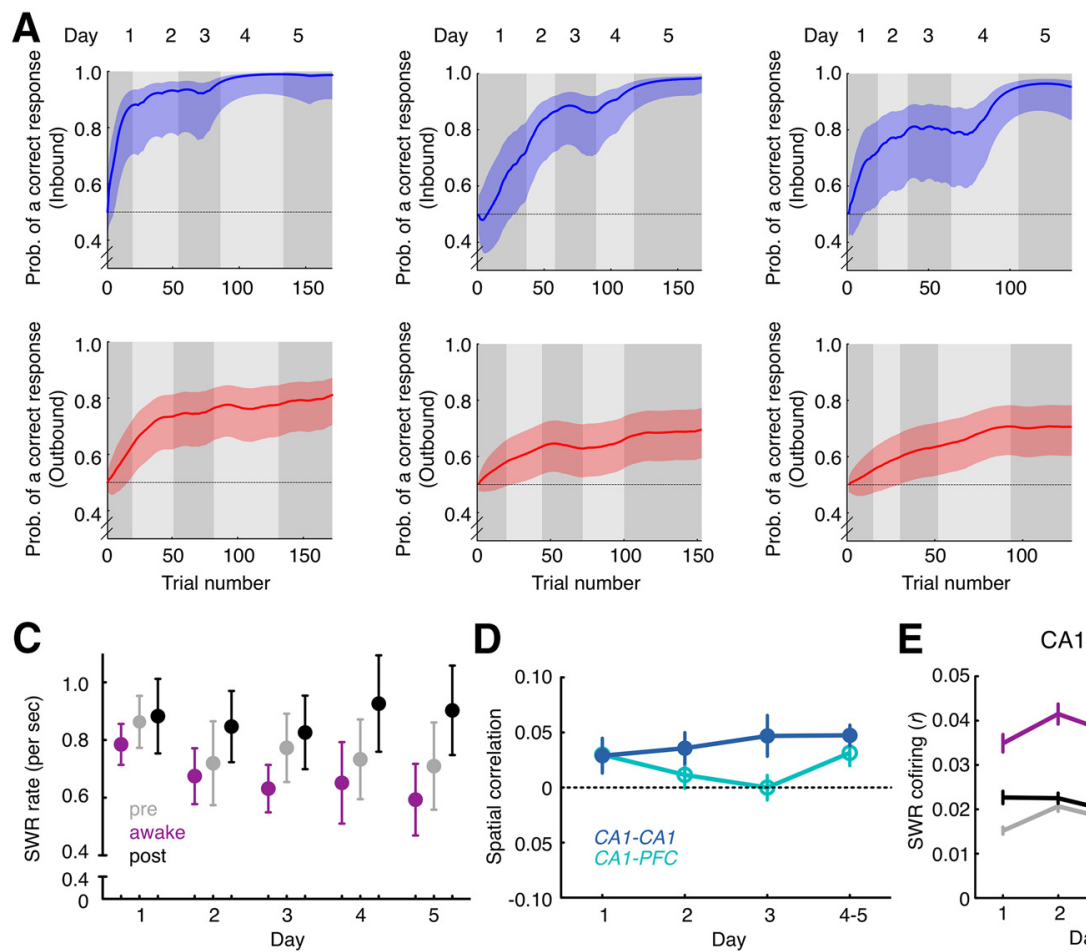

H

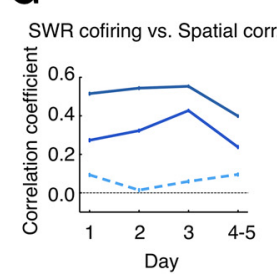

CA1-CA1

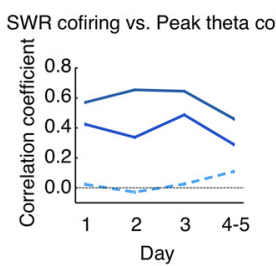

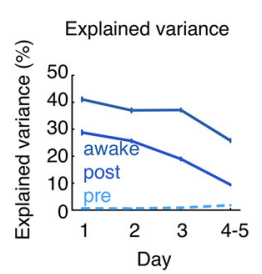

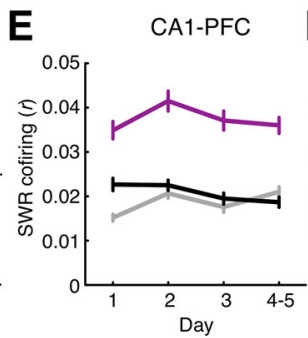

B

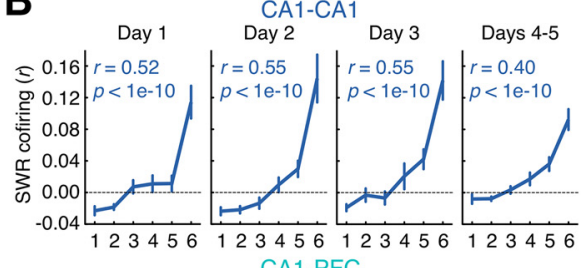

CA1-PFC

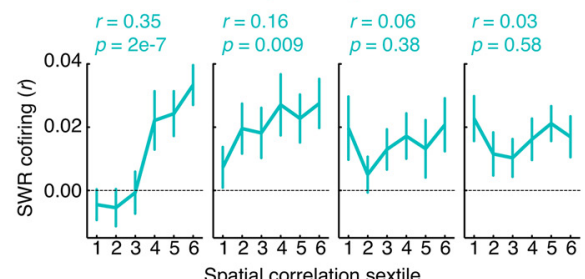

CA1-CA1
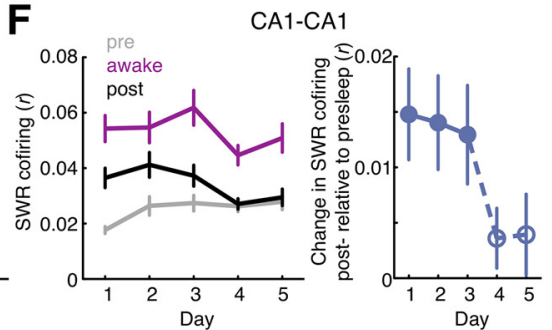

CA1-PFC

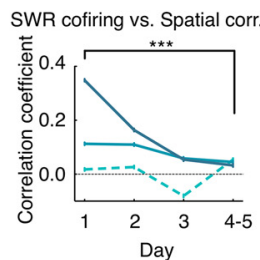

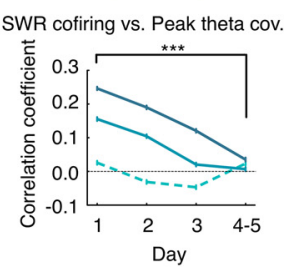

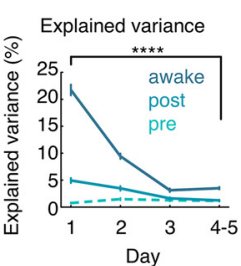

Figure 7. Changes in SWR reactivation over multiday learning. $A$, Behavior performance of all three multiday animals. Top, Inbound learning curves. Bottom, Outbound learning curves. Learning curves are estimated using a state-space model (Jadhav et al., 2012). Shadings, 90\% confidence intervals; horizontal dashed lines, chance-level performance of 0.5 . B, Awake-SWR co-firing plotted as a function of spatial correlation across days for CA1-CA1 (top; $n=239,221,198,552$ ) and CA1-PFC (bottom; $n=246,269,251,297$ ) pairs. Note that there is a reduction in spatial reactivation over learning, especially for CA1-PFC pairs $(Z=3.82$ and $p=0.0001$ for day 1 vs days $4-5)$. Error bars, SEM. C, SWR rate during wakefulness and SWS/ NREM across days. D, Spatial correlation of spatial firing maps of cell pairs across days. Overall spatial correlation did not change over days ( $p=0.169$ and 0.171 for 1762 CA1-CA1 and 1897 CA1-PFC pairs, respectively; Kruskal-Wallis tests). $\boldsymbol{E}$, SWR co-firing of CA1-PFC cell pairs across days. SWR co-firing was stronger during awake SWRs throughout learning ( $p$ values $<0.0001$, rank sum tests). The number of pairs is noted in $B$. $F$, Left, SWR co-firing of CA1-CA1 cell pairs across days. Right, Change in SWR co-firing of CA1-CA1 pairs from pre-task sleep to post-task sleep. The change in SWR co-firing from pre-task to post-task sleep diminished with experience, similar to that reported previously ( $0^{\prime}$ Neill et al., 2008). Closed and open circles, respectively, indicate changes significantly $(p=0.013,0.045,0.025$ for days $1-3$, respectively) and nonsignificantly ( $p=0.12$ and 0.11 for days 4,5 , respectively; rank sum tests) different from zero. The number of pairs for each day is noted in $\boldsymbol{B}$. $\boldsymbol{G}, \boldsymbol{H}$, Spearman correlation coefficient between SWR co-firing versus spatial correlation (left), or SWR co-firing versus peak theta covariance (middle), and explained variance during SWRs (right), for CA1-CA1 (G) and CA1-PFC $(\boldsymbol{H})$ pairs across days. Note the reduction in CA1-PFC reactivation over days. Error bars represent bootstrapped $95 \%$ confidence intervals $\left(n=500\right.$ times). ${ }^{* * *} p$ values $<0.001, z$ tests for comparing correlation coefficients; ${ }^{* * *} p<0.0001$, bootstrapping for explained variance, $n=500$ times. Note that the small error bars may not be discernable in some cases. All $95 \%$ Cl error bars are, however, plotted. Data are from three multiday animals.

relationship between reactivation and learning, we examined changes in reactivation by combining both time scales in our data, multiday and single-day learning. We quantified reactivation at different points in learning by combining data from the same learning stages across the two time scales (Fig. 8), similar to a previous study (Singer et al., 2013). Reactivation was compared in the following epochs corresponding to different learning stages: during initial exposure to the novel environment, first session where animals performed above-chance (initial learning), first session where animals performed $>75 \%$ correct (later learning), and the last session in which animals performed asymptotically $>75 \%$ correct (Fig. 8A). An earlier study showed that CA1 reactivation had the most prominent influence on decision-making behavior during initial and later learning stages (Singer et al., 2013). We found that CA1 reactivation remained significant across all learning stages (Fig. 8 B, C; see Materials and Meth- ods), as expected from the observation of significant reactivation across all days in Figure 7G. For CA1-PFC reactivation (Fig. $8 D, E$ ), however, we found a significant increase in reactivation from the session with first exposure (with highest novelty) to the initial learning session (Fig. $8 E ; p=0.0026$ and $1 \mathrm{e}-8$ for first session and initial learning, respectively; $p=$ 0.0001 , comparing first exposure and initial learning, $p$ values are from bootstrapping, $n=500$ times), followed by a subsequent decrease to nonsignificant reactivation during asymptomatic performance ( $p=0.053$ and 0.134 for later learning and asymptomatic performance, respectively; $p$ values are from bootstrapping, $n=500$ times). This increase in reactivation from the first exposure to the initial learning session, despite decrease in novelty, suggests a relationship between CA1-PFC reactivation and behavioral learning beyond just a novelty effect. 

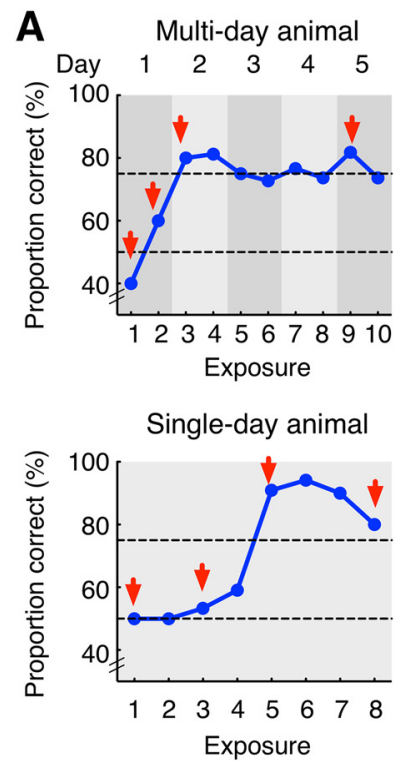

B

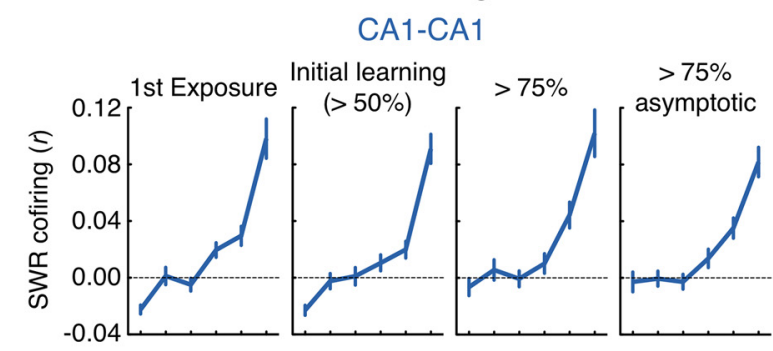

D

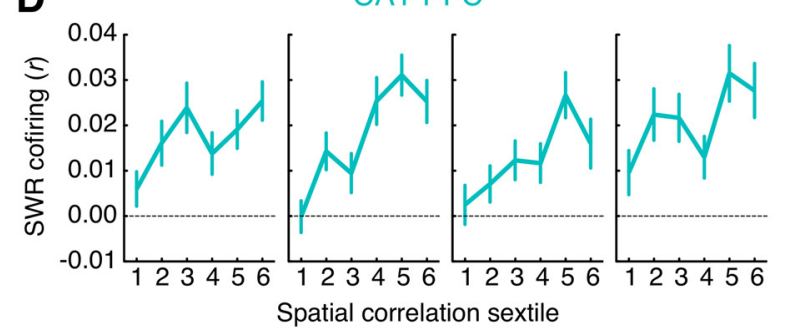

C

CA1-CA1

SWR cofiring vs. Spatial corr.
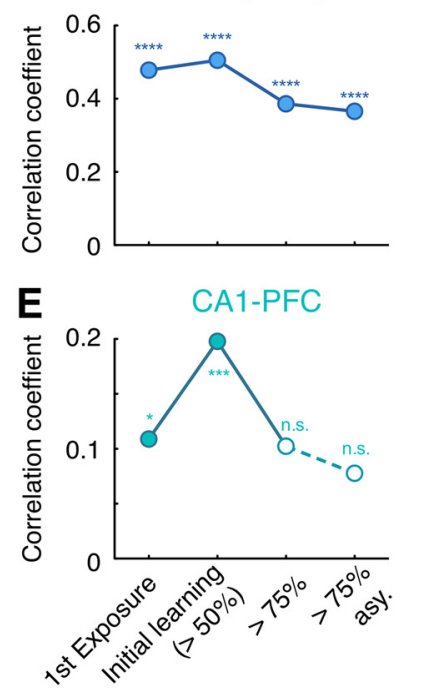

Figure 8. CA1-PFC reactivation is enhanced during initial learning. A, Proportion correct on outbound trials per exposure session for one multiday and one single-day animals. Sessions corresponding to performance categories 1-4 (see Materials and Methods) are indicated by red arrowheads. Horizontal dashed lines: 50\% and 75\% correct. B, Awake-SWR co-firing plotted as a function of spatial correlation across performance categories for CA1-CA1 pairs $(n=543,585,481,649)$. Error bars, SEM. C, Spearman correlation coefficient between awake-SWR co-firing versus spatial correlation for CA1-CA1 pairs shown in $\boldsymbol{B}$ across performance categories. $\boldsymbol{D}$, Awake-SWR co-firing plotted as a function of spatial correlation across performance categories for CA1-PFC cell pairs $(n=762,823,819,713)$. Error bars, SEM. $\boldsymbol{E}$, Spearman correlation coefficient between awake-SWR co-firing versus spatial correlation for CA1-PFC pairs shown in $\boldsymbol{D}$ across performance categories. Open circles and dashed lines indicate non-significant correlation $(p>0.05)$. Data are from three animals that learned the task over $5 d$, and two animals that learned over multiple sessions in a single day. ${ }^{*} p<0.05,{ }^{* * *} p<0.001,{ }^{* * * *} p<0.0001$, n.S., $p>0.05 ; p$ values are from bootstrapping, $n=500$ times.

\section{Discussion}

Our results establish marked differences in hippocampal-prefrontal reactivation during awake and sleep SWRs, and demonstrate that awake reactivation is a stronger and more structured representation of behavioral experiences during spatial learning. We found that individual prefrontal neurons responded differently during awake versus sleep SWRs, leading to a lack of correlation in modulation patterns. Temporal coordination of CA1-PFC spiking activity was stronger during awake SWRs, and spatial reactivation in the hippocampal-prefrontal network was significantly enhanced during awake versus sleep SWRs. This structured awake reactivation during behavior was observed despite a lack of coordination between hippocampal ripples and cortical delta and spindle oscillations that is seen in sleep. Finally, awake hippocampal-prefrontal reactivation was significantly enhanced during initial learning in novel environments.

Reactivation during awake and sleep SWRs has been of particular interest because of their hypothesized roles in memory processes, and because both have been causally linked to learning; disrupting hippocampal activity during both awake and sleep SWRs impairs learning in spatial memory tasks (Girardeau et al., 2009; Ego-Stengel and Wilson, 2010; Jadhav et al., 2012). Whereas sleep SWRs have a proposed role in memory consolidation, awake SWRs are thought to play a role not just in memory formation, but also retrieval, planning, and prospective behavior (Carr et al., 2011; Roumis and Frank, 2015). Although SWRs occur in these two distinct states: awake SWRs, which primarily occur during brief pauses in exploratory behavior, and sleep SWRs in continuous stretches of slow-wave (nonREM) sleep disjoint from behavior, the relationship between the two forms of reactivation during behavioral learning remained unclear.
Our experiments were designed to quantify the relationship between reactivation in these two states using simultaneous recordings during sleep and behavior over the course of spatial learning. We found a surprising lack of correlation in prefrontal modulation patterns during awake and sleep SWRs. This difference is remarkable given that both are thought to reactivate recent experiences. Whereas prefrontal neurons showed excitation and inhibition in equivalent proportions during awake SWRs, sleep SWRs were dominated by excitatory modulation. Inhibition of individual prefrontal neurons was best explained by immobility coding of locations in the current environment where SWRs occur prominently, either reward locations on the W-track where animals pause between trials, or nesting positions in the rest box. This suggests that inhibitory patterns are primarily determined by immobility coding in the current environment, with reduction in inhibitory modulation during sleep SWRs possibly due to the limited number of nesting positions in the rest box. The lack of correlation in modulation across the entire population is indicative that CA1-PFC reactivation will be different for awake versus sleep SWRs.

Indeed, we found that temporal coordination in CA1-PFC network was enhanced during awake SWRs. The average timing of modulation was also different, with a propensity for PFC excitation during sleep to occur earlier relative to SWR onset, indicating this could provide contextual input that biases CA1 replay events (Wang and Ikemoto, 2016; Rothschild et al., 2017). CA1PFC neuron pairs showed more synchronized co-firing, indicating better timing coordination during awake reactivation. Spatial reactivation was also enhanced during awake SWRs relative to post-task sleep SWRs; pairwise measures comparing spatial correlation to SWR co-firing showed stronger reactivation for awake SWRs. Further, for repeated run-sleep sessions during learning, 
this enhanced reactivation persisted for awake SWRs regardless of the relative order of sleep sessions. Interestingly, we did not see a preservation of timing relationships during reactivation in CA1-PFC pairs, unlike in CA1 (Karlsson and Frank, 2009). A previous study also reported reactivation of behavioral experiences as synchronously firing PFC cell assemblies occurring during hippocampal sleep SWRs (Peyrache et al., 2009). Synchronization of cell assemblies, observed in both CA1 and PFC (Kudrimoti et al., 1999; Peyrache et al., 2009; van de Ven et al., 2016), may thus offer a better measure of coordinated hippocampal-prefrontal reactivation during initial learning and performance. This ensemble synchronization measure also showed that awake CA1-PFC reactivation was a significantly more accurate representation of behavioral experiences in the current task than sleep reactivation.

The reactivation differences led us to examine cortical network oscillations associated with awake versus sleep SWRs. Coordination of cortical delta and spindle oscillations with SWRs is thought to be a key feature of sleep reactivation, and has also been shown to contribute to memory consolidation (Sirota et al., 2003; Peyrache et al., 2011; Maingret et al., 2016). We found that this coordination was significantly reduced during awake SWRs, indicating that there is a fundamental difference in brain-wide network patterns for the two cases. The nested oscillations in sleep, which include thalamocortical and hippocampal networks, provide coordination and spread of synchronous activity across large-scale networks necessary for consolidation, and may especially be required for integrating activity related to multiple experiences for building schemas and for general inference, suggested functions of sleep in memory (McClelland et al., 1995; Marshall and Born, 2007; Tse et al., 2011; Battaglia et al., 2012; Tamminen et al., 2013; Roumis and Frank, 2015; Penagos et al., 2017). Whereas during awake states, more precise reactivation of the current task-related experience may be necessary for accurate representations of ongoing behavioral variables to inform memoryguided decisions (Karlsson and Frank, 2009; O'Neill et al., 2010; Carr et al., 2011; Yu and Frank, 2015; Ambrose et al., 2016; Papale et al., 2016; Wu et al., 2017). The difference in network oscillations is a key indicator of the different roles that awake and sleep SWRs may play in memory.

Finally, how do these reactivation processes relate to learning? Both awake and sleep reactivation within hippocampus have been reported to show dynamics related to experience and learning. Sleep SWRs play a role in consolidation of CA1 cell assemblies in novel, but not familiar environments (van de Ven et al., 2016). Awake CA1 reactivation is stronger in novel environments (Cheng and Frank, 2008), supports memory-guided choices in spatial tasks (Singer et al., 2013; Papale et al., 2016), and is also linked to place field stability during learning (Roux et al., 2017). Here, we directly examined how measures of CA1 and CA1-PFC reactivation changed over learning in the novel $\mathrm{W}$-track task. CA1 reactivation decreased with experience, but stayed significant for both awake and sleep SWRs even as animals achieved stable performance. CA1-PFC reactivation was significantly elevated during initial learning and was strongest for awake SWRs, but rapidly declined with experience. This decline in reactivation with experience paralleled both, a decrease in novelty and an increase in behavioral learning. A role in initial learning is also supported by the observation of a small but significant increase in CA1-PFC reactivation from the first exposure on the track (uncharted novelty) to the session that first reached above-chance performance (initial learning). Therefore, CA1-PFC reactivation can be regulated by novelty exposure and may have a role in behavioral learning, as suggested for CA1 reactivation (Singer et al., 2013). The reactivation trends over learning suggest that awake SWRs may play a key role in stabilization of task-related variables and establishing functional links across long-range networks during initial learning. Awake SWRs are already linked to place field stability during learning (Dupret et al., 2013; Roux et al., 2017), and our results raise the intriguing possibility that this stabilization role may not just be limited to hippocampus, but extend to prefrontal areas for building a coherent cognitive map to support learning.

One limitation of the current study is that the measures of reactivation were biased toward spatial activity, and it is possible that non-spatial measures such as rule-related representations may also be independently reactivated during SWRs, and show different trends from spatial reactivation. This possibility can be addressed in future experiments that examine reactivation during multiple learning tasks, or in non-spatial tasks. Another possibility to note is that different stages within NREM sleep have been proposed to support various stages of consolidation, including potentiation and homeostasis (Genzel et al., 2014). A recent study also reported independent reactivation in entorhinal cortex during sleep outside SWRs (O'Neill et al., 2017). Thus, independent physiological processes during NREM sleep, not measured in our study, could also additionally contribute to consolidation.

The most parsimonious explanation of our results is that awake hippocampal-prefrontal reactivation is a more structured representation of ongoing experiences that can support multiple functions required for memory-guided behavior: retrieval, planning, evaluation, and prospection. In addition, accurate awake reactivation may be required to drive stabilization of representations during initial learning in novel environments. In contrast, sleep reactivation may play a broader role than just consolidation of recent experiences, possibly integrating activity from multiple experiences in widespread networks during consolidation to build schemas, for general inference and semantic knowledge. The coordination of network oscillations during sleep may be a key feature for such integration, and widespread activation may result in nonspecific excitation, leading to less accurate reactivation for a recent behavioral experience. These findings thus provide a necessary foundation for future studies to investigate the complementary and overlapping functions of awake and sleep hippocampal-cortical reactivation in learning and memory-guided behavior.

\section{References}

Ambrose RE, Pfeiffer BE, Foster DJ (2016) Reverse replay of hippocampal place cells is uniquely modulated by changing reward. Neuron 91:11241136. CrossRef Medline

Battaglia FP, Benchenane K, Sirota A, Pennartz CM, Wiener SI (2011) The hippocampus: hub of brain network communication for memory. Trends Cogn Sci 15:310-318. CrossRef Medline

Battaglia FP, Borensztajn G, Bod R (2012) Structured cognition and neural systems: from rats to language. Neurosci Biobehav Rev 36:1626-1639. CrossRef Medline

Bendor D, Wilson MA (2012) Biasing the content of hippocampal replay during sleep. Nat Neurosci 15:1439-1444. CrossRef Medline

Buzsáki G (1996) The hippocampo-neocortical dialogue. Cereb Cortex 6:81-92. CrossRef Medline

Buzsáki G (2015) Hippocampal sharp wave-ripple: a cognitive biomarker for episodic memory and planning. Hippocampus 25:1073-1188. CrossRef Medline

Carr MF, Jadhav SP, Frank LM (2011) Hippocampal replay in the awake state: a potential substrate for memory consolidation and retrieval. Nat Neurosci 14:147-153. CrossRef Medline

Cheng S, Frank LM (2008) New experiences enhance coordinated neural activity in the hippocampus. Neuron 57:303-313. CrossRef Medline 
Colgin LL (2016) Rhythms of the hippocampal network. Nat Rev Neurosci 17:239-249. CrossRef Medline

Diekelmann S, Born J (2010) The memory function of sleep. Nat Rev Neurosci 11:114-126. CrossRef Medline

Dragoi G, Tonegawa S (2011) Preplay of future place cell sequences by hippocampal cellular assemblies. Nature 469:397-401. CrossRef Medline

Dupret D, O’Neill J, Csicsvari J (2013) Dynamic reconfiguration of hippocampal interneuron circuits during spatial learning. Neuron 78:166180. CrossRef Medline

Ego-Stengel V, Wilson MA (2010) Disruption of ripple-associated hippocampal activity during rest impairs spatial learning in the rat. Hippocampus 20:1-10. CrossRef Medline

Eichenbaum H, Cohen NJ (2001) From conditioning to conscious recollection. New York: Oxford UP.

Eschenko O, Ramadan W, Mölle M, Born J, Sara SJ (2008) Sustained increase in hippocampal sharp-wave ripple activity during slow-wave sleep after learning. Learn Mem 15:222-228. CrossRef Medline

Euston DR, Tatsuno M, McNaughton BL (2007) Fast-forward playback of recent memory sequences in prefrontal cortex during sleep. Science 318 : 1147-1150. CrossRef Medline

Foster DJ, Wilson MA (2006) Reverse replay of behavioural sequences in hippocampal place cells during the awake state. Nature 440:680-683. CrossRef Medline

Genzel L, Kroes MC, Dresler M, Battaglia FP (2014) Light sleep versus slow wave sleep in memory consolidation: a question of global versus local processes? Trends Neurosci 37:10-19. CrossRef Medline

Girardeau G, Benchenane K, Wiener SI, Buzsáki G, Zugaro MB (2009) Selective suppression of hippocampal ripples impairs spatial memory. Nat Neurosci 12:1222-1223. CrossRef Medline

Grosmark AD, Buzsáki G (2016) Diversity in neural firing dynamics supports both rigid and learned hippocampal sequences. Science 351:14401443. CrossRef Medline

Gupta AS, van der Meer MA, Touretzky DS, Redish AD (2010) Hippocampal replay is not a simple function of experience. Neuron 65:695-705. CrossRef Medline

Inostroza M, Born J (2013) Sleep for preserving and transforming episodic memory. Annu Rev Neurosci 36:79-102. CrossRef Medline

Jadhav SP, Kemere C, German PW, Frank LM (2012) Awake hippocampal sharp-wave ripples support spatial memory. Science 336:1454-1458. CrossRef Medline

Jadhav SP, Rothschild G, Roumis DK, Frank LM (2016) Coordinated excitation and inhibition of prefrontal ensembles during awake hippocampal sharp-wave ripple events. Neuron 90:113-127. CrossRef Medline

Jarosiewicz B, McNaughton BL, Skaggs WE (2002) Hippocampal population activity during the small-amplitude irregular activity state in the rat. J Neurosci 22:1373-1384. Medline

Ji D, Wilson MA (2007) Coordinated memory replay in the visual cortex and hippocampus during sleep. Nat Neurosci 10:100-107. CrossRef Medline

Karlsson MP, Frank LM (2009) Awake replay of remote experiences in the hippocampus. Nat Neurosci 12:913-918. CrossRef Medline

Kay K, Sosa M, Chung JE, Karlsson MP, Larkin MC, Frank LM (2016) A hippocampal network for spatial coding during immobility and sleep. Nature 531:185-190. CrossRef Medline

Kudrimoti HS, Barnes CA, McNaughton BL (1999) Reactivation of hippocampal cell assemblies: effects of behavioral state, experience, and EEG dynamics. J Neurosci 19:4090-4101. Medline

Logothetis NK, Eschenko O, Murayama Y, Augath M, Steudel T, Evrard HC, Besserve M, Oeltermann A (2012) Hippocampal-cortical interaction during periods of subcortical silence. Nature 491:547-553. CrossRef Medline

Maingret N, Girardeau G, Todorova R, Goutierre M, Zugaro M (2016) Hippocampo-cortical coupling mediates memory consolidation during sleep. Nat Neurosci 19:959-964. CrossRef Medline

Marshall L, Born J (2007) The contribution of sleep to hippocampusdependent memory consolidation. Trends Cogn Sci 11:442-450. CrossRef Medline

Marshall L, Helgadóttir H, Mölle M, Born J (2006) Boosting slow oscillations during sleep potentiates memory. Nature 444:610-613. CrossRef Medline

McClelland JL, McNaughton BL, O’Reilly RC (1995) Why there are complementary learning systems in the hippocampus and neocortex: insights from the successes and failures of connectionist models of learning and memory. Psychol Rev 102:419-457. CrossRef Medline

Miyawaki H, Diba K (2016) Regulation of hippocampal firing by network oscillations during sleep. Curr Biol 26:893-902. CrossRef Medline

Mizuseki K, Diba K, Pastalkova E, Buzsáki G (2011) Hippocampal CA1 pyramidal cells form functionally distinct sublayers. Nat Neurosci 14:11741181. CrossRef Medline

Ólafsdóttir HF, Barry C, Saleem AB, Hassabis D, Spiers HJ (2015) Hippocampal place cells construct reward related sequences through unexplored space. eLife 4:e06063. CrossRef Medline

O’Neill J, Senior TJ, Allen K, Huxter JR, Csicsvari J (2008) Reactivation of experience-dependent cell assembly patterns in the hippocampus. Nat Neurosci 11:209-215. CrossRef Medline

O'Neill J, Pleydell-Bouverie B, Dupret D, Csicsvari J (2010) Play it again: reactivation of waking experience and memory. Trends Neurosci 33:220 229. CrossRef Medline

O’Neill J, Boccara CN, Stella F, Schoenenberger P, Csicsvari J (2017) Superficial layers of the medial entorhinal cortex replay independently of the hippocampus. Science 355:184-188. CrossRef Medline

Papale AE, Zielinski MC, Frank LM, Jadhav SP, Redish AD (2016) Interplay between hippocampal sharp-wave-ripple events and vicarious trial and error behaviors in decision making. Neuron 92:975-982. CrossRef Medline

Penagos H, Varela C, Wilson MA (2017) Oscillations, neural computations and learning during wake and sleep. Curr Opin Neurobiol 44:193-201. CrossRef Medline

Peyrache A, Khamassi M, Benchenane K, Wiener SI, Battaglia FP (2009) Replay of rule-learning related neural patterns in the prefrontal cortex during sleep. Nat Neurosci 12:919-926. CrossRef Medline

Peyrache A, Benchenane K, Khamassi M, Wiener SI, Battaglia FP (2010) Principal component analysis of ensemble recordings reveals cell assemblies at high temporal resolution. J Comput Neurosci 29:309-325. CrossRef Medline

Peyrache A, Battaglia FP, Destexhe A (2011) Inhibition recruitment in prefrontal cortex during sleep spindles and gating of hippocampal inputs. Proc Natl Acad Sci U S A 108:17207-17212. CrossRef Medline

Pfeiffer BE, Foster DJ (2013) Hippocampal place-cell sequences depict future paths to remembered goals. Nature 497:74-79. CrossRef Medline

Rasch B, Büchel C, Gais S, Born J (2007) Odor cues during slow-wave sleep prompt declarative memory consolidation. Science 315:1426-1429. CrossRef Medline

Rothschild G, Eban E, Frank LM (2017) A cortical-hippocampal-cortical loop of information processing during memory consolidation. Nat Neurosci 20:251-259. CrossRef Medline

Roumis DK, Frank LM (2015) Hippocampal sharp-wave ripples in waking and sleeping states. Curr Opin Neurobiol 35:6-12. CrossRef Medline

Roux L, Hu B, Eichler R, Stark E, Buzsáki G (2017) Sharp wave ripples during learning stabilize the hippocampal spatial map. Nat Neurosci 20: 845-853. CrossRef Medline

Sejnowski TJ, Destexhe A (2000) Why do we sleep? Brain Res 886:208-223. CrossRef Medline

Shin JD, Jadhav SP (2016) Multiple modes of hippocampal-prefrontal interactions in memory-guided behavior. Curr Opin Neurobiol 40:161169. CrossRef Medline

Siapas AG, Wilson MA (1998) Coordinated interactions between hippocampal ripples and cortical spindles during slow-wave sleep. Neuron 21:1123-1128. CrossRef Medline

Siapas AG, Lubenov EV, Wilson MA (2005) Prefrontal phase locking to hippocampal theta oscillations. Neuron 46:141-151. CrossRef Medline

Singer AC, Frank LM (2009) Rewarded outcomes enhance reactivation of experience in the hippocampus. Neuron 64:910-921. CrossRef Medline

Singer AC, Carr MF, Karlsson MP, Frank LM (2013) Hippocampal SWR activity predicts correct decisions during the initial learning of an alternation task. Neuron 77:1163-1173. CrossRef Medline

Sirota A, Csicsvari J, Buhl D, Buzsáki G (2003) Communication between neocortex and hippocampus during sleep in rodents. Proc Natl Acad Sci U S A 100:2065-2069. CrossRef Medline

Staresina BP, Bergmann TO, Bonnefond M, van der Meij R, Jensen O, Deuker L, Elger CE, Axmacher N, Fell J (2015) Hierarchical nesting of slow oscillations, spindles and ripples in the human hippocampus during sleep. Nat Neurosci 18:1679-1686. CrossRef Medline 
Sutherland GR, McNaughton B (2000) Memory trace reactivation in hippocampal and neocortical neuronal ensembles. Curr Opin Neurobiol 10: 180-186. CrossRef Medline

Tamminen J, Lambon Ralph MA, Lewis PA (2013) The role of sleep spindles and slow-wave activity in integrating new information in semantic memory. J Neurosci 33:15376-15381. CrossRef Medline

Tse D, Takeuchi T, Kakeyama M, Kajii Y, Okuno H, Tohyama C, Bito H, Morris RG (2011) Schema-dependent gene activation and memory encoding in neocortex. Science 333:891-895. CrossRef Medline

van de Ven GM, Trouche S, McNamara CG, Allen K, Dupret D (2016) Hippocampal offline reactivation consolidates recently formed cell assembly patterns during sharp wave-ripples. Neuron 92:968-974. CrossRef Medline

Wang DV, Ikemoto S (2016) Coordinated interaction between hippocam- pal sharp-wave ripples and anterior cingulate unit activity. J Neurosci 36:10663-10672. CrossRef Medline

Wierzynski CM, Lubenov EV, Gu M, Siapas AG (2009) State-dependent spike-timing relationships between hippocampal and prefrontal circuits during sleep. Neuron 61:587-596. CrossRef Medline

Wilson MA, McNaughton BL (1994) Reactivation of hippocampal ensemble memories during sleep. Science 265:676-679. CrossRef Medline

Wiltgen BJ, Brown RA, Talton LE, Silva AJ (2004) New circuits for old memories: the role of the neocortex in consolidation. Neuron 44:101108. CrossRef Medline

Wu CT, Haggerty D, Kemere C, Ji D (2017) Hippocampal awake replay in fear memory retrieval. Nat Neurosci 20:571-580. CrossRef Medline

Yu JY, Frank LM (2015) Hippocampal-cortical interaction in decision making. Neurobiol Learn Mem 117:34-41. CrossRef Medline 